\title{
Stationary Oldroyd model with diffusive stress in thin pipes
}

\author{
Laurent Chupin, Andrei Ichim and Sébastien Martin*
}

February 3, 2017

\begin{abstract}
The aim of this paper is to study the stationary flow of an Oldroyd fluid with particular diffusive stress, flowing through a thin 3D pipe. An asymptotic expansion with respect to a small parameter describing the ratio between the diameter of the cross section and the length is calculated and rigorously justified. The result obtained is compared against a numerical solution in the particular case of axisymmetric geometries.
\end{abstract}

\section{Introduction}

We consider the stationary flow of an incompressible Oldroyd fluid with diffusive stress in a thin tube - these equations could model the flow of blood or other substances in the human body since it is known that such biological fluids exhibit non-newtonian behaviour. The word "thin" is used to express the fact that the typical diameter of such pipe is much smaller when compared to its length - mathematically we shall use a small parameter $\varepsilon$ to express this ratio. Note that in the standard Oldroyd model the diffusive stress term is usually omitted, since it is orders of magnitude smaller than the other terms in the equation. Taking the aforementioned facts into account, we suppose that this term is of order $\varepsilon^{2}$ - while there is no physical motivation for this choice, we argue that it is needed to obtain a "good" matching of asymptotic coefficients in a sense we shall indicate later.

Firstly, we prove the existence of the solution for our problem - its uniqueness will be shown later under additional hypothesis on $\varepsilon$. In terms of actually finding this solution, since the full $3 \mathrm{D}$ model is very difficult to deal with, we shall discuss two ways of reducing its complexity. The first method is the same as the one used in [6]: it involves writing the asymptotic expansion of the rescaled solution with respect to $\varepsilon$, formally computing its coefficients and finally showing the validity of this expansion by proving a convergence result. The second possibility is to reduce the $3 \mathrm{D}$ problem to a $2 \mathrm{D}$ one in the particular case of an axis-symmetric domain. Two numerical algorithms for solving this reduced problem are presented, and the corresponding solutions are compared to the one obtained via the first method.

We conclude this section with some bibliographical remarks. The first mathematical investigation of the Oldroyd model was done by Renardy, who proved an existence result in the stationary case in [12]. On the subject of the asymptotic behaviour of Newtonian fluids in thin pipes there are many results by E. Mariušić-Paloka, see for instance [9], and G. Panasenko [11], among others. A more recent paper on quasi-newtonian fluids in thin pipes is [6].

\section{Statement of the problem}

The equations describing the model presented in the previous section can be written as a system coupling the velocity $u$, the pressure $p$ and the extra-stress $\sigma$ :

$$
\left\{\begin{aligned}
-(1-r) \Delta u+\operatorname{Re}(u \cdot \nabla) \cdot u+\nabla p & =\operatorname{div} \sigma+f_{\varepsilon} \\
\operatorname{divu} & =0 \\
\operatorname{We}\left((u \cdot \nabla) \sigma+g_{a}(\sigma, \nabla u)\right)+\sigma-\varepsilon^{2} \Delta \sigma & =2 r D u
\end{aligned}\right.
$$

where

$$
g_{a}(\sigma, \nabla u)=(\sigma \cdot W u-W u \cdot \sigma)+a(\sigma \cdot D u+D u \cdot \sigma)
$$

*Université Paris Descartes, Laboratoire MAP5 (CNRS UMR 8145), 45 rue des Saints-Pères, 75270 Paris cedex 06, France, sebastien.martin@parisdescartes.fr 
with

$$
W u=\frac{\nabla u-(\nabla u)^{T}}{2}, \quad D u=\frac{\nabla u+(\nabla u)^{T}}{2} .
$$

By $f_{\varepsilon}$ we have denoted the exterior forces acting on the fluid. We have used Re $>0$ the Reynolds number, We $>0$ the Weissenberg number, $r \in(0,1)$ the retardation parameter, and $a \in[-1,1]$. The parameter $\varepsilon$ will be assume a "small" positive number. It appears in the diffusive coefficient for the stress but it is linked to the geometry of the domain $\Omega_{\varepsilon}$ where the fluid flows:

$$
\Omega_{\varepsilon}=\left\{\left(x_{1}, x_{2}, x_{3}\right) \in \mathbb{R}^{3} \mid x_{1} \in(0,1),\left(x_{2}, x_{3}\right) \in \varepsilon S\left(x_{1}\right)\right\},
$$

where $S\left(x_{1}\right)$ are sufficiently regular domains - without insisting too much, we also suppose the application $x_{1} \mapsto S\left(x_{1}\right)$ is also smooth enough $\left(C^{2}\right.$ regularity is sufficient). For the sake of simplicity we are going to assume $\Omega_{\varepsilon} \subset[0,1] \times[0, \varepsilon] \times[0, \varepsilon]$, with $0<\varepsilon \leq 1$, and the three-part boundary of $\Omega_{\varepsilon}$ is given by the following:

$$
\begin{aligned}
& \Gamma_{\varepsilon}=\left\{\left(x_{1}, x_{2}, x_{3}\right) \in \mathbb{R}^{3} \mid x_{1} \in(0,1),\left(x_{2}, x_{3}\right) \in \varepsilon \partial S\left(x_{1}\right)\right\} \\
& \Sigma_{0}^{\varepsilon}=\varepsilon S(0) \text { and } \Sigma_{1}^{\varepsilon}=\varepsilon S(1) .
\end{aligned}
$$

Since we intend to use periodic boundary conditions, we shall assume that the domain is "cylindrical" near the boundaries, that is to say $S\left(x_{1}\right)=S(0)=S(1)=S\left(1-x_{1}\right)$ for $x_{1} \in(0, \delta), \delta$ being a any positive real.

As for the boundary conditions we consider

$$
\begin{cases}u=0 \text { on } \Gamma_{\varepsilon}, & \\ \sigma=0 \text { on } \Gamma_{\varepsilon}, & \\ u_{\mid \Sigma_{0}^{\varepsilon}}=u_{\mid \Sigma_{1}^{\varepsilon}}, & \sigma_{\mid \Sigma_{0}^{\varepsilon}}=\sigma_{\mid \Sigma_{1}^{\varepsilon}}, \\ \partial_{1} u_{\mid \Sigma_{0}^{\varepsilon}}=\partial_{1} u_{\mid \Sigma_{1}^{\varepsilon}}, & \partial_{1} \sigma_{\mid \Sigma_{0}^{\varepsilon}}=\partial_{1} \sigma_{\mid \Sigma_{1}^{\varepsilon}}, \\ p_{\mid \Sigma_{0}^{\varepsilon}}-p_{\mid \Sigma_{1}^{\varepsilon}}=p_{d}, & \end{cases}
$$

where the constant $p_{d}$ corresponds to the given pressure difference at the two ends of the tube.

For the remainder of this paper, we will make two assumptions:

1. The first assumption is about the parameter $a \in[-1,1]$ introduced in order to describe different objective (frame indifferent) time derivatives. For technical reason, we will assume

$$
a=0 .
$$

From a physical point of view, this choice corresponds to the Jaumann (or co-rotational) derivative. This restriction is used to obtain the existence of a stationary solution in Section 3. Except for this theoretical section, it seems that the more general case $a \neq 0$ can be treated in the same way. In particular, the asymptotic model obtained in the case $\varepsilon \rightarrow 0$ is independent of the value of $a$.

2. The second assumption relates to the force term $f_{\varepsilon}$. We assume that it satisfies one of the following conditions:

(F1) There exist smooth $\tilde{f}: \Omega_{\varepsilon} \rightarrow \mathbb{R}^{3}$ such that $f_{\varepsilon}\left(x_{1}, x_{k}\right)=\tilde{f}\left(x_{1}, \frac{x_{k}}{\varepsilon}\right)$.

(F2) $\sup _{\varepsilon}\left\|f_{\varepsilon}\right\|_{L^{\infty}\left(\Omega_{\varepsilon}\right)}<\infty$.

Finally, let us conclude with some remarks on notations. We shall denote $\Omega=\Omega_{1}, \Gamma=\Gamma_{1}$. We will call a function $v: \Omega_{\varepsilon} \rightarrow \mathbb{R}^{k}$ smooth whenever $v \in C^{2}\left(\overline{\Omega_{\varepsilon}}\right)$. Henceforth, $\|\cdot\|$ will mean the $L^{2}$ norm on $\Omega_{\varepsilon}$, while $\|\cdot\|_{0}$ will be used for the $L^{2}$ norm on $\Omega$. We shall denote by $C$ various constants independent of $\varepsilon$.

\section{The existence problem}

The proof of the existence of a weak solution is based on classical energy estimates and compactness arguments. We proceed very carefully to show that some boundary conditions are verified in a very weak sense. Defining

$$
H_{\#}^{1}\left(\Omega_{\varepsilon}\right)=\left\{v \in H^{1}\left(\Omega_{\varepsilon}\right) \mid v_{\mid \Sigma_{0}^{\varepsilon}}=v_{\mid \Sigma_{1}^{\varepsilon}}\right\}
$$


we consider the following spaces

$$
\begin{aligned}
& V=\left\{v \in\left(H_{\#}^{1}\left(\Omega_{\varepsilon}\right)\right)^{3} \mid \operatorname{div} v=0, v=0 \quad \text { on } \quad \Gamma_{\varepsilon}\right\}, \\
& W=\left\{\tau \in \mathbb{M}_{3 \times 3}\left(H_{\#}^{1}\left(\Omega_{\varepsilon}\right)\right) \mid \tau_{i j}=\tau_{j i}, \tau=0 \quad \text { on } \Gamma_{\varepsilon}\right\} .
\end{aligned}
$$

The variational problem can be written as:

$$
\left\{\begin{array}{l}
\text { Find }(u, \sigma) \in V \times W \text { such that, for all }(v, \tau) \in V \times W, \\
(1-r) \int_{\Omega_{\varepsilon}} \nabla u: \nabla v+\operatorname{Re} \int_{\Omega_{\varepsilon}}(u \cdot \nabla) u \cdot v+\int_{\Omega_{\varepsilon}} \sigma: D v=\int_{\Omega_{\varepsilon}} f_{\varepsilon} v+p_{d} \int_{\Sigma_{0}^{\varepsilon}} v_{1}, \\
\operatorname{We} \int_{\Omega_{\varepsilon}}\left((u \cdot \nabla) \sigma+g_{0}(\sigma, \nabla u)\right): \tau+\int_{\Omega_{\varepsilon}} \sigma: \tau+\varepsilon^{2} \int_{\Omega_{\varepsilon}} \nabla \sigma: \nabla \tau=2 r \int_{\Omega_{\varepsilon}} D u: \tau .
\end{array}\right.
$$

Evidently, every classical solution

$$
(u, \sigma, p) \in\left(C^{2}\left(\Omega_{\varepsilon}\right) \cap C^{1}\left(\overline{\Omega_{\varepsilon}}\right)\right) \times\left(C^{2}\left(\Omega_{\varepsilon}\right) \cap C^{1}\left(\overline{\Omega_{\varepsilon}}\right)\right) \times\left(C^{1}\left(\Omega_{\varepsilon}\right) \cap C\left(\overline{\Omega_{\varepsilon}}\right)\right)
$$

which verifies (1) and (2) is a solution to (3). The goal is to prove that there exists a solution to the problem (3), which is also a so-called weak solution to the problem (1)-(2).

Proposition 1. For all $\varepsilon>0$ the problem (3) admits at least one solution $(u, \sigma) \in V \times W$, which satisfies

$$
\|\nabla u\| \leq C \varepsilon^{2}, \quad\|\nabla \sigma\| \leq C \varepsilon .
$$

Proof. We consider the Hilbert space $V \times W$ endowed with the scalar product:

$$
\left\langle\left(u_{1}, \sigma_{1}\right),\left(u_{2}, \sigma_{2}\right)\right\rangle=\left(u_{1}, u_{2}\right)_{V}+\varepsilon^{2}\left(\sigma_{1}, \sigma_{2}\right)_{W},
$$

where

$$
\left(u_{1}, u_{2}\right)_{V}=\int_{\Omega_{\varepsilon}} \nabla u_{1}: \nabla u_{2}, \quad\left(\sigma_{1}, \sigma_{2}\right)_{W}=\int_{\Omega_{\varepsilon}} \nabla \sigma_{1}: \nabla \sigma_{2} .
$$

Let $\left\{v_{1}, \ldots, v_{n}, \ldots\right\}$ be a dense basis in $V,\left\{\tau_{1}, \ldots, \tau_{n}, \ldots\right\}$ a dense basis in $W$, and let $V_{n}, W_{n}$ be the subspaces generated by $\left\{v_{1}, \ldots, v_{n}\right\}$ and $\left\{\tau_{1}, \ldots, \tau_{n}\right\}$ respectively. We show that the approximate problem:

"Find $\left(u_{n}, \sigma_{n}\right) \in V_{n} \times W_{n}$ such that (3) holds for all $(v, \tau) \in V_{n} \times W_{n} "$

has a solution satisfying (4). We first define the application $P_{n}: V_{n} \times W_{n} \rightarrow V_{n} \times W_{n}$ by

$$
\begin{aligned}
\left\langle P_{n}(u, \sigma),(v, \tau)\right\rangle= & \operatorname{r}\left((1-r) \int_{\Omega_{\varepsilon}} \nabla u: \nabla v+\operatorname{Re} \int_{\Omega_{\varepsilon}}(u \cdot \nabla) u \cdot v+\int_{\Omega_{\varepsilon}} \sigma: D u-\int_{\Omega_{\varepsilon}} f_{\varepsilon} v-p_{d} \int_{\Sigma_{0}^{\varepsilon}} v_{1}\right) \\
& +\left(\operatorname{We} \int_{\Omega_{\varepsilon}}\left((u \cdot \nabla) \sigma+g_{0}(\sigma, \nabla u)\right): \tau+\int_{\Omega_{\varepsilon}} \sigma: \tau+\varepsilon^{2} \int_{\Omega_{\varepsilon}} \nabla \sigma: \nabla \tau-2 r \int_{\Omega_{\varepsilon}} D u: \tau\right) .
\end{aligned}
$$

For any $(u, \sigma) \in V_{n} \times W_{n}$, we evaluate $\left\langle P_{n}(u, \sigma),(u, \sigma)\right\rangle$ as follows

$$
\left\langle P_{n}(u, \sigma),(u, \sigma)\right\rangle=2 r(1-r)\|\nabla u\|^{2}-2 r \int_{\Omega_{\varepsilon}} f_{\varepsilon} u-2 r p_{d} \int_{\Sigma_{0}^{\varepsilon}} u_{1}+\|\sigma\|^{2}+\varepsilon^{2}\|\nabla \sigma\|^{2} .
$$

To obtain previous equality, we have used the classical following cancellations (which are due to the free-divergence of the velocity, the homogeneous Dirichlet boundary condition for the velocity, and due to the symmetry of the stress tensor):

$$
\int_{\Omega_{\varepsilon}}(u \cdot \nabla) u \cdot u=\int_{\Omega_{\varepsilon}}(u \cdot \nabla) \sigma: \sigma=\int_{\Omega_{\varepsilon}}(\sigma \cdot W u-W u \cdot \sigma): \sigma=0 .
$$

Before passing further, let us recall an elementary result: 
Lemma 1. Let $u \in H^{1}\left(\Omega_{\varepsilon}\right)$ with $u=0$ on $\Gamma_{\varepsilon}$. Then

$$
\|u\| \leq \varepsilon\|\nabla u\|, \quad\|u\|_{L^{4}} \leq c_{0} \varepsilon^{1 / 4}\|\nabla u\| .
$$

Moreover, for all $u \in V$ we have

$$
\left|\int_{\Sigma_{0}^{\varepsilon}} u_{1}\right| \leq \varepsilon^{2}\|\nabla u\|
$$

Proof. See for instance [6].

Returning to the proof of Proposition 1 we can then write

$$
\left|p_{d} \int_{\Omega_{\varepsilon}} u_{1}\right| \leq p_{d} \varepsilon^{2}\|\nabla u\|
$$

whereas the term $\int_{\Omega_{\varepsilon}} f_{\varepsilon} u$ is treated differently depending on the assumption on the source $f_{\varepsilon}$.

- In the case where the assumption (F1) holds, we have

$$
\left|\int_{\Omega_{\varepsilon}} f_{\varepsilon} u\right| \leq\left\|f_{\varepsilon}\right\|\|u\| \leq \varepsilon\|f\|_{0} \cdot \varepsilon\|\nabla u\|
$$

- In the case where the assumption (F2) holds, we write

$$
\begin{aligned}
\left|\int_{\Omega_{\varepsilon}} f_{\varepsilon} u\right| & \leq \sup _{\varepsilon}\left\|f_{\varepsilon}\right\|_{L^{\infty}\left(\Omega_{\varepsilon}\right)} \int_{\Omega_{\varepsilon}}|u| \leq \sup _{\varepsilon}\left\|f_{\varepsilon}\right\|_{L^{\infty}\left(\Omega_{\varepsilon}\right)}\left|\Omega_{\varepsilon}\right|^{1 / 2}\|u\| \\
& \leq \sup _{\varepsilon}\left\|f_{\varepsilon}\right\|_{L^{\infty}\left(\Omega_{\varepsilon}\right)} \cdot \varepsilon \cdot \varepsilon\|\nabla u\| .
\end{aligned}
$$

In both cases, denoting $\xi=(u, \sigma)$, and $\|\xi\|^{2}=\|\nabla u\|^{2}+\varepsilon^{2}\|\nabla \sigma\|^{2}$, we deduce from the equation (5) that

$$
\left\langle P_{n}(\xi), \xi\right\rangle \geq 2 r(1-r)\|\xi\|^{2}-2 r \varepsilon^{2}\left(C_{f}+p_{d}\right)\|\xi\|,
$$

the constant $C_{f}$ being defined by $C_{f}=\|f\|_{0}$ in the case (F1), and by $C_{f}=\sup _{\varepsilon}\left\|f_{\varepsilon}\right\|_{L^{\infty}\left(\Omega_{\varepsilon}\right)}$ in the case (F2). Consequently, for

$$
\|\xi\|=\frac{C_{f}+p_{d}}{1-r} \varepsilon^{2}
$$

we have $\left\langle P_{n}(\xi), \xi\right\rangle \geq 0$. Since $P_{n}$ is clearly continuous, using a well known result - see, for instance, [7] - we derive the existence of $\left(u_{n}, \sigma_{n}\right) \in V_{n} \times W_{n}$ such that $P_{n}\left(u_{n}, \sigma_{n}\right)=0$, and, in addition, it follows easily

$$
\left\|\nabla u_{n}\right\| \leq \frac{C_{f}+p_{d}}{1-r} \varepsilon^{2}, \quad\left\|\nabla \sigma_{n}\right\| \leq \frac{C_{f}+p_{d}}{1-r} \varepsilon .
$$

Since $P_{n}\left(u_{n}, \sigma_{n}\right)$ was constructed as a sum of two linear independent functionals, it follows that $\left(u_{n}, \sigma_{n}\right)$ is a solution to the approximate problem above mentioned. Using (6) and the compact embedding of $H^{1}$ into $L^{4}$ we have, up to a subsequence,

$$
\begin{array}{ll}
u_{m} \rightarrow u & \text { weakly in } V, \\
\sigma_{m} \rightarrow \sigma & \text { weakly in } W, \\
u_{m} \rightarrow u & \text { strongly in } L^{4}, \\
\sigma_{m} \rightarrow \sigma & \text { strongly in } L^{4} .
\end{array}
$$

It is immediate that the limit $(u, \sigma)$ satisfy $(4)$; we show next that $(u, \sigma)$ is the desired solution. Fix $n \geq 1$, and $(v, \tau) \in V_{n} \times W_{n}$. Then for $m \geq n$ (in the above subsequence sense), we can write

$$
\left\{\begin{array}{l}
(1-r) \int_{\Omega_{\varepsilon}} \nabla u_{m}: \nabla v+\operatorname{Re} \int_{\Omega_{\varepsilon}}\left(u_{m} \cdot \nabla\right) u_{m} \cdot v+\int_{\Omega_{\varepsilon}} \sigma_{m}: D v=\int_{\Omega_{\varepsilon}} f_{\varepsilon} v+p_{d} \int_{\Sigma_{0}^{\varepsilon}} v_{1}, \\
\operatorname{We} \int_{\Omega_{\varepsilon}}\left(\left(u_{m} \cdot \nabla\right) \sigma_{m}+g_{0}\left(\sigma_{m}, \nabla u_{m}\right)\right): \tau+\int_{\Omega_{\varepsilon}} \sigma_{m}: \tau+\varepsilon^{2} \int_{\Omega_{\varepsilon}} \nabla \sigma_{m}: \nabla \tau=2 r \int_{\Omega_{\varepsilon}} D\left(u_{m}\right): \tau
\end{array}\right.
$$


To prove the convergence, let us write the following estimates

$$
\begin{aligned}
\left|\int_{\Omega_{\varepsilon}}\left(u_{m} \cdot \nabla\right) u_{m} \cdot v-\int_{\Omega_{\varepsilon}}(u \cdot \nabla) u \cdot v\right| & \leq \int_{\Omega_{\varepsilon}}\left|\left(\left(u_{m}-u\right) \cdot \nabla\right) u_{m} \cdot v\right|+\int_{\Omega_{\varepsilon}}\left|(u \cdot \nabla)\left(u_{m}-u\right) \cdot v\right| \\
& \leq\left\|u_{m}-u\right\|_{L^{4}}\left\|\nabla u_{m}\right\|\left\|\left|\|_{L^{4}}+\int_{\Omega_{\varepsilon}}\right|(u \cdot \nabla)\left(u_{m}-u\right) \cdot v \mid,\right. \\
\left|\int_{\Omega_{\varepsilon}}\left(u_{m} \cdot \nabla\right) \sigma_{m}: \tau-\int_{\Omega_{\varepsilon}}(u \cdot \nabla) \sigma: \tau\right| & \leq \int_{\Omega_{\varepsilon}}\left|\left(\left(u_{m}-u\right) \cdot \nabla\right) \sigma_{m}: \tau\right|+\int_{\Omega_{\varepsilon}}\left|(u \cdot \nabla)\left(\sigma_{m}-\sigma\right): \tau\right| \\
& \leq\left\|u_{m}-u\right\|_{L^{4}}\left\|\nabla \sigma_{m}\right\|\|\tau\|_{L^{4}}+\int_{\Omega_{\varepsilon}}\left|(u \cdot \nabla)\left(\sigma_{m}-\sigma\right): \tau\right|, \\
\left|\int_{\Omega_{\varepsilon}} g_{0}\left(\sigma_{m}, \nabla u_{m}\right): \tau-\int_{\Omega_{\varepsilon}} g_{0}(\sigma, \nabla u): \tau\right| & \leq \int_{\Omega_{\varepsilon}}\left|g_{0}\left(\sigma_{m}-\sigma, \nabla u_{m}\right): \tau\right|+\int_{\Omega_{\varepsilon}}\left|g_{0}\left(\sigma, \nabla\left(u_{m}-u\right)\right): \tau\right| \\
& \leq\left\|\sigma_{m}-\sigma\right\|_{L^{4}}\left\|\nabla u_{m}\right\|\|\tau\|_{L^{4}}+\int_{\Omega_{\varepsilon}}\left|g_{0}\left(\sigma, \nabla\left(u_{m}-u\right)\right): \tau\right| .
\end{aligned}
$$

Owing to (7a)-(7d) and the continuous embedding of $H^{1}$ into $L^{4}$, we easily derive that all the terms on the left hand side are convergent to 0 . The remaining linear terms are trivial. We readily derive that $(u, \sigma)$ is a solution to $(3)$.

Next we show that the solution whose existence was proven at the first step is in fact a weak solution for the problem (1)-(2).

Proposition 2. For all $\varepsilon>0$, the solution $(u, \sigma)$ to the variational problem (3) allows to obtain a weak solution $(u, p, \sigma) \in$ $V \times L^{2}\left(\Omega_{\varepsilon}\right) \times W$ to the problem $(1)-(2)$.

Proof. Since $V^{\prime}=\left\{v \in\left(C_{0}^{\infty}\left(\Omega_{\varepsilon}\right)\right)^{3}\right.$, div $\left.v=0\right\} \subset V$ and $W^{\prime}=\mathbb{M}_{3 \times 3}\left(C_{0}^{\infty}\left(\Omega_{\varepsilon}\right)\right) \subset W$ it easily follows from (3) that, for all $v \in V^{\prime}, w \in W^{\prime}$,

$$
\left\{\begin{array}{l}
\left\langle-(1-r) \Delta u+\operatorname{Re}(u \cdot \nabla) \cdot u-\operatorname{div} \sigma-f_{\varepsilon}-\overline{p_{d}}, v\right\rangle=0, \\
\left\langle\operatorname{We}((u \cdot \nabla) \sigma+g(\sigma, \nabla u))+\sigma-\varepsilon^{2} \Delta \sigma-2 r D u, \tau\right\rangle=0,
\end{array}\right.
$$

where the brackets understood in the distributional sense. Moreover, the overline $\overline{p_{d}} \in V^{\prime}$ is given by $\left\langle\overline{p_{d}}, v\right\rangle=p_{d} \int_{\Sigma_{0}^{\varepsilon}} v_{1}$. A simple application of the de Rham theory enables us to retrieve the pressure $p \in L^{2}\left(\Omega_{\varepsilon}\right)$ such that (1) holds in the distributional sense. To conclude we need to verify the last two boundary conditions in (2). We shall show only those involving $u$ and $p$, since the remaining one (involving $\sigma$ ) is derived exactly in the same way. At first glance it looks strange, since $L^{2}$ functions don't have boundary trace, but we shall see the exact sense in which these equalities are understood. First, let us introduce the following result:

Lemma 2. If $f \in\left(L^{2}\left(\Omega_{\varepsilon}\right)\right)^{3}$ such that div $f \in L^{\frac{3}{2}}\left(\Omega_{\varepsilon}\right)$. Then $f \cdot n \in H^{-\frac{1}{2}}\left(\partial \Omega_{\varepsilon}\right)$ in the following sense

$$
\langle f \cdot n, \phi\rangle=\int_{\Omega_{\varepsilon}}(\phi \operatorname{div} f+f \cdot \nabla \phi), \quad \forall \phi \in H^{1}\left(\Omega_{\varepsilon}\right)
$$

(where $\langle\cdot, \cdot\rangle$ denotes the pairing between $H^{-\frac{1}{2}}\left(\partial \Omega_{\varepsilon}\right)$ and $\left.H^{\frac{1}{2}}\left(\partial \Omega_{\varepsilon}\right)\right)$ meaning that the term on the right hand side is only dependent on the boundary trace value of $\phi$, and that it defines a linear continuous functional on $H^{\frac{1}{2}}\left(\partial \Omega_{\varepsilon}\right)$.

Proof. Using a result in [4] we can find a $g \in\left(W^{1, \frac{3}{2}}\left(\Omega_{\varepsilon}\right)\right)^{3}$ such that

$$
\operatorname{div} g=\operatorname{div} f
$$

From [10] we get that

$$
\int_{\partial \Omega_{\varepsilon}} \phi g \cdot n=\int_{\Omega_{\varepsilon}}(\phi \operatorname{div} g+g \cdot \nabla \phi), \quad \forall \phi \in H^{1}\left(\Omega_{\varepsilon}\right) .
$$

By using the continuous inclusion of $H^{\frac{1}{2}}\left(\partial \Omega_{\varepsilon}\right)$ into $L^{3}\left(\partial \Omega_{\varepsilon}\right)$, it is immediate that the term on the left hand side defines a linear continuous functional on $H^{\frac{1}{2}}\left(\partial \Omega_{\varepsilon}\right)$. 
Next, by standard Sobolev embeddings, we have $g \in\left(W^{1, \frac{3}{2}}\left(\Omega_{\varepsilon}\right)\right)^{3} \subset\left(L^{2}\left(\Omega_{\varepsilon}\right)\right)^{3}$ and so $f-g \in\left(L^{2}\left(\Omega_{\varepsilon}\right)\right)^{3}$, while div $(f-$ $g)=0 \in\left(L^{2}\left(\Omega_{\varepsilon}\right)\right)^{3}$. Hence by using the result in [5] we derive $(f-g) \cdot n \in H^{-\frac{1}{2}}\left(\partial \Omega_{\varepsilon}\right)$ and

$$
\langle(f-g) \cdot n, \phi\rangle=\int_{\Omega_{\varepsilon}}(f-g) \cdot \nabla \phi, \quad \forall \phi \in H^{1}\left(\Omega_{\varepsilon}\right) .
$$

Hence, for $h \in H^{\frac{1}{2}}\left(\partial \Omega_{\varepsilon}\right)$, then given $\phi_{h} \in H^{1}\left(\Omega_{\varepsilon}\right)$ such that $\phi_{h}=h$ on $\partial \Omega_{\varepsilon}$, the application

$$
h \mapsto \int_{\Omega_{\varepsilon}}\left(\phi_{h} \operatorname{div} f+f \cdot \nabla \phi_{h}\right)
$$

is well defined and determines a linear continuous functional on $H^{\frac{1}{2}}\left(\partial \Omega_{\varepsilon}\right)$, being the sum of two applications enjoying this property. The proof of the lemma 2 is then completed.

In order to achieve the proof of Proposition 2, observe that for $i \in\{1,2,3\}$, we have (the vectors $e_{i}$ being the vectors of the usual basis of $\mathbb{R}^{3}$ )

$$
\operatorname{div}\left(-(1-r) \nabla u_{i}+p e_{i}\right)=(\operatorname{div} \sigma+f-u \cdot \nabla u)_{i} \in L^{\frac{3}{2}}\left(\Omega_{\varepsilon}\right),
$$

and since $-(1-r) \nabla u_{i}+p e_{i} \in\left(L^{2}\left(\Omega_{\varepsilon}\right)\right)^{3}$ we can use Lemma 2 to get $\left(-(1-r) \nabla u_{i}+p e_{i}\right) \cdot n \in H^{-\frac{1}{2}}\left(\partial \Omega_{\varepsilon}\right)$ and, for all $\phi \in H^{1}\left(\Omega_{\varepsilon}\right)$ and all $i \in\{1,2,3\}$ :

$$
\left\langle\left(-(1-r) \nabla u_{i}+p e_{i}\right) \cdot n, \phi\right\rangle=\int_{\Omega_{\varepsilon}}\left((\operatorname{div} \sigma+f-u \cdot \nabla u)_{i} \phi-(1-r) \nabla u_{i} \nabla \phi+p \partial_{i} \phi\right) .
$$

Let $h \in H^{\frac{1}{2}}\left(\Sigma_{0}^{\varepsilon}\right)=H^{\frac{1}{2}}\left(\Sigma_{1}^{\varepsilon}\right)$, and let

$$
h^{j}= \begin{cases}h e_{j} & \text { on } \Sigma_{0}^{\varepsilon} \cup \Sigma_{0}^{\varepsilon}, \\ 0 & \text { on } \Gamma_{\varepsilon} .\end{cases}
$$

It is clear that $h^{j} \in H^{\frac{1}{2}}\left(\partial \Omega_{\varepsilon}\right)$ and $\int_{\partial \Omega_{\varepsilon}} h^{j}=0$. Using a known result [4], we find $v^{j} \in V$ such that div $v^{j}=0$ and $v^{j}=h^{j}$ on $\partial \Omega_{\varepsilon}$, for all $j \in\{1,2,3\}$. By taking in (8) test functions $v_{i}^{j}$, summing for $i \in\{1,2,3\}$, and taking into account that $u$ is a variational solution we get, for any $j \in\{1,2,3\}$ :

$$
\left\langle\left(-(1-r) \nabla u_{j}+p e_{j}\right) \cdot n, h\right\rangle_{0}+\left\langle\left(-(1-r) \nabla u_{j}+p e_{j}\right) \cdot n, h\right\rangle_{1}=-p_{d} \delta_{1 j} \int_{\Sigma_{0}^{\varepsilon}} h,
$$

where $\langle\cdot, \cdot\rangle_{k}$ denotes the pairing between $H^{-\frac{1}{2}}\left(\Sigma_{k}^{\varepsilon}\right)$ and $H^{\frac{1}{2}}\left(\Sigma_{k}^{\varepsilon}\right)$, for $k \in\{0,1\}$. Taking into account that $n=-e_{1}$ on $\Sigma_{0}^{\varepsilon}$ and $n=e_{1}$ on $\Sigma_{1}^{\varepsilon}$, it follows that $\left.\partial_{1} u_{k}\right|_{\Sigma_{0}^{\varepsilon}}=\left.\partial_{1} u_{k}\right|_{\Sigma_{1}^{\varepsilon}}$ for $k \in\{2,3\}$ and that

$$
\left\langle-(1-r) \partial_{1} u_{1}+p, h\right\rangle_{0}-\left\langle-(1-r) \partial_{1} u_{1}+p, h\right\rangle_{1}=p_{d} \int_{\Sigma_{0}^{\varepsilon}} h .
$$

To conclude the proof, we only need to only that $\left.\partial_{1} u_{1}\right|_{\Sigma_{0}^{\varepsilon}}=\left.\partial_{1} u_{1}\right|_{\Sigma_{1}^{\varepsilon}}$. First, observe that $\partial_{1} u \in L^{2}\left(\Omega_{\varepsilon}\right)$ and $\operatorname{div}\left(\partial_{1} u\right)=0$, so that, by Lemma $2, \partial_{1} u \cdot n \in H^{-\frac{1}{2}}(\partial \Omega)$ and

$$
\left\langle\partial_{1} u \cdot n, \phi\right\rangle=\int_{\Omega_{\varepsilon}} \partial_{1} u \cdot \nabla \phi, \quad \forall \phi \in H^{1}(\Omega) .
$$

Take any $\phi \in C^{2}\left(\overline{\Omega_{\varepsilon}}\right)$ such that $\left.\phi\right|_{\Sigma_{0}^{\varepsilon}}=\left.\phi\right|_{\Sigma_{1}^{\varepsilon}}$. We have

$$
\begin{aligned}
\int_{\Omega_{\varepsilon}} \partial_{1} u \cdot \nabla \phi & =-\int_{\Omega_{\varepsilon}} u \cdot \nabla \partial_{1} \phi+\int_{\partial \Omega_{\varepsilon}} u \cdot \nabla \phi n_{1} \\
& =\int_{\Omega_{\varepsilon}} \operatorname{div} u \partial_{1} \phi-\int_{\partial \Omega_{\varepsilon}} \partial_{1} \phi u \cdot n+\int_{\partial \Omega_{\varepsilon}} u \cdot \nabla \phi n_{1},
\end{aligned}
$$

but, since $u \in V$ and $n_{2}=n_{3}=0$ on $\Sigma_{0}^{\varepsilon}, \Sigma_{1}^{\varepsilon}$ we get

$$
\left\langle\partial_{1} u_{1}, \phi\right\rangle_{0}-\left\langle\partial_{1} u_{1}, \phi\right\rangle_{1}=\int_{\partial \Omega_{\varepsilon}} \partial_{2} \phi u_{2}+\partial_{3} \phi u_{3}=0
$$

using that $u \in V$ and the periodicity of $\phi$; hence, by a density argument we obtain the desired result.

The uniqueness of this solution will be proved later under additional hypothesis on $\varepsilon$. 


\section{Asymptotic expansion}

From now on, we are going to assume that $f_{\varepsilon}$ satisfies condition (F1). Moreover, we will also systematically use the dummy index $k$ to represent either summation for $k \in\{2,3\}$, either the repeated index.

Let $(u, p, \sigma)$ be a solution of (1) and (2), and consider the applications $\widetilde{u}: \Omega \rightarrow \mathbb{R}^{3}, \widetilde{\sigma}: \Omega \rightarrow \mathbb{M}_{3 \times 3}(\mathbb{R})$ and $\widetilde{p}: \Omega \rightarrow \mathbb{R}$ defined by

$$
\begin{aligned}
& \widetilde{u}\left(x_{1}, x_{k}\right)=u\left(x_{1}, \varepsilon x_{k}\right), \\
& \widetilde{\sigma}\left(x_{1}, x_{k}\right)=\sigma\left(x_{1}, \varepsilon x_{k}\right), \\
& \widetilde{p}\left(x_{1}, x_{k}\right)=p\left(x_{1}, \varepsilon x_{k}\right) .
\end{aligned}
$$

By a-priori estimates, we easily derive

$$
\|\widetilde{u}\|_{0} \leq C \varepsilon^{2}, \quad\|\widetilde{\sigma}\|_{0} \leq C \varepsilon .
$$

So let us formally write

$$
\begin{aligned}
& \widetilde{u}_{1}=\varepsilon^{2} \widetilde{u}_{1}^{0}+\cdots+\varepsilon^{2 n+2} \widetilde{u}_{1}^{n}+\ldots \\
& \widetilde{u}_{k}=\varepsilon^{3} \widetilde{u}_{k}^{0}+\cdots+\varepsilon^{2 n+3} \widetilde{u}_{k}^{n}+\ldots \\
& \widetilde{p}=\widetilde{p}^{0}+\cdots+\varepsilon^{2 n} \widetilde{p}^{n}+\ldots \\
& \widetilde{\sigma}_{11}=\varepsilon^{2} \widetilde{\sigma}_{11}^{0}+\cdots+\varepsilon^{2 n+2} \widetilde{\sigma}_{11}^{n}+\ldots \\
& \widetilde{\sigma}_{1 k}=\varepsilon \widetilde{\sigma}_{1 k}^{0}+\cdots+\varepsilon^{2 n+1} \widetilde{\sigma}_{1 k}^{n}+\ldots \\
& \widetilde{\sigma}_{\ell m}=\varepsilon^{2} \widetilde{\sigma}_{\ell m}^{0}+\cdots+\varepsilon^{2 n+2} \widetilde{\sigma}_{\ell m}^{n}+\ldots
\end{aligned}
$$

for $\ell, m \in\{2,3\}$. Obviously, we assume that all $\widetilde{u}_{\alpha}^{m}, \widetilde{\sigma}_{\alpha \beta}^{m}, \widetilde{p}^{m}$ are independent of $\varepsilon$ for all $m \geq 0, \alpha, \beta \in\{1,2,3\}$. Upon writing the equations in the rescaled domain $\Omega$, plugging in the expansions above and identifying the appropriate powers of $\varepsilon$ we get the following 10 equations for each $j \geq 0$ (we have tacitly assumed that terms with negative upper coefficients are 0 ):

- Each component of the momentum equation:

$$
\begin{aligned}
& -(1-r) \partial_{k}^{2} \widetilde{u}_{1}^{j}+\partial_{1} \widetilde{p}^{j}=\partial_{1} \widetilde{\sigma}_{11}^{j-1}+\partial_{k} \widetilde{\sigma}_{1 k}^{j}+\widetilde{h}_{1}^{j} \\
& -(1-r) \partial_{k}^{2} \widetilde{u}_{2}^{j}+\partial_{2} \widetilde{p}^{j+1}=\partial_{1} \widetilde{\sigma}_{12}^{j}+\partial_{k} \widetilde{\sigma}_{2 k}^{j}+\widetilde{h}_{2}^{j} \\
& -(1-r) \partial_{k}^{2} \widetilde{u}_{3}^{j}+\partial_{3} \widetilde{p}^{j+1}=\partial_{1} \widetilde{\sigma}_{13}^{j}+\partial_{k} \widetilde{\sigma}_{3 k}^{j}+\widetilde{h}_{3}^{j}
\end{aligned}
$$

where

$$
\begin{aligned}
& \widetilde{h}_{1}^{j}=(1-r) \partial_{1}^{2} \widetilde{u}_{1}^{j-1}-\operatorname{Re} \sum_{i=0}^{j-2}\left(\widetilde{u}_{1}^{i} \partial_{1} \widetilde{u}_{1}^{j-i-2}+\widetilde{u}_{k}^{i} \partial_{k} \widetilde{u}_{1}^{j-i-2}\right)+\widetilde{f}_{1}^{j} \\
& \widetilde{h}_{2}^{j}=(1-r) \partial_{1}^{2} \widetilde{u}_{2}^{j-1}-\operatorname{Re} \sum_{i=0}^{j-3}\left(\widetilde{u}_{1}^{i} \partial_{1} \widetilde{u}_{2}^{j-i-3}+\widetilde{u}_{k}^{i} \partial_{k} \widetilde{u}_{2}^{j-i-3}\right)+\widetilde{f}_{2}^{j} \\
& \widetilde{h}_{3}^{j}=(1-r) \partial_{1}^{2} \widetilde{u}_{3}^{j-1}-\operatorname{Re} \sum_{i=0}^{j-3}\left(\widetilde{u}_{1}^{i} \partial_{1} \widetilde{u}_{3}^{j-i-3}+\widetilde{u}_{k}^{i} \partial_{k} \widetilde{u}_{3}^{j-i-3}\right)+\widetilde{f}_{3}^{j}
\end{aligned}
$$

with $\widetilde{f}_{\alpha}^{j}=\widetilde{f}_{\alpha}$ for $j=0$ and $\widetilde{f}_{\alpha}^{j}=0$ for $j>0$.

- The six components of the symmetric stress given by the Oldroyd model:

$$
\begin{aligned}
\mathrm{We}\left[\sum_{i=0}^{j-1}\left(\widetilde{u}_{\alpha}^{i} \partial_{\alpha} \widetilde{\sigma}_{11}^{j-i-1}-\partial_{1} \widetilde{u}_{k}^{i} \widetilde{\sigma}_{1 k}^{j-i-1}\right)\right. & \left.+\sum_{i=0}^{j} \partial_{k} \widetilde{u}_{1}^{i} \widetilde{\sigma}_{1 k}^{j-i}\right] \\
& +\widetilde{\sigma}_{11}^{j}-\partial_{1}^{2} \widetilde{\sigma}_{11}^{j-1}-\partial_{k}^{2} \widetilde{\sigma}_{11}^{j}=2 r \partial_{1} \widetilde{u}_{1}^{j}
\end{aligned}
$$




$$
\begin{aligned}
& \mathrm{We}\left[\sum_{i=0}^{j-1} \widetilde{u}_{\alpha}^{i} \partial_{\alpha} \widetilde{\sigma}_{12}^{j-i-1}+\frac{1}{2} \sum_{i=0}^{j-2}\left(\partial_{1} \widetilde{u}_{2}^{i} \widetilde{\sigma}_{11}^{j-i-2}-\partial_{1} \widetilde{u}_{k}^{i} \widetilde{\sigma}_{2 k}^{j-i-2}\right)\right. \\
& \left.+\frac{1}{2} \sum_{i=0}^{j-1}\left(-\partial_{2} \widetilde{u}_{1}^{i} \widetilde{\sigma}_{11}^{j-i-1}+\left(\partial_{3} \widetilde{u}_{2}^{i}-\partial_{2} \widetilde{u}_{3}^{i}\right) \widetilde{\sigma}_{13}^{j-i-1}+\partial_{k} \widetilde{u}_{1}^{i} \widetilde{\sigma}_{2 k}^{j-i-1}\right)\right] \\
& +\widetilde{\sigma}_{12}^{j}-\partial_{1}^{2} \widetilde{\sigma}_{12}^{j-1}-\partial_{k}^{2} \widetilde{\sigma}_{12}^{j}=r\left(\partial_{1} \widetilde{u}_{2}^{j-1}+\partial_{2} \widetilde{u}_{1}^{j}\right) \\
& \mathrm{We}\left[\sum_{i=0}^{j-1} \widetilde{u}_{\alpha}^{i} \partial_{\alpha} \widetilde{\sigma}_{13}^{j-i-1}+\frac{1}{2} \sum_{i=0}^{j-2}\left(\partial_{1} \widetilde{u}_{3}^{i} \widetilde{\sigma}_{11}^{j-i-2}-\partial_{1} \widetilde{u}_{k}^{i} \widetilde{\sigma}_{3 k}^{j-i-2}\right)\right. \\
& \left.+\frac{1}{2} \sum_{i=0}^{j-1}\left(-\partial_{3} \widetilde{u}_{1}^{i} \widetilde{\sigma}_{11}^{j-i-1}+\left(\partial_{2} \widetilde{u}_{3}^{i}-\partial_{3} \widetilde{u}_{2}^{i}\right) \widetilde{\sigma}_{12}^{j-i-1}+\partial_{k} \widetilde{u}_{1}^{i} \widetilde{\sigma}_{3 k}^{j-i-1}\right)\right] \\
& +\widetilde{\sigma}_{13}^{j}-\partial_{1}^{2} \widetilde{\sigma}_{13}^{j-1}-\partial_{k}^{2} \widetilde{\sigma}_{13}^{j}=r\left(\partial_{1} \widetilde{u}_{3}^{j-1}+\partial_{3} \widetilde{u}_{1}^{j}\right) \\
& \mathrm{We}\left[\sum_{i=0}^{j-1}\left(\widetilde{u}_{\alpha}^{i} \partial_{\alpha} \widetilde{\sigma}_{22}^{j-i-1}+\partial_{1} \widetilde{u}_{2}^{i} \widetilde{\sigma}_{12}^{j-i-1}+\partial_{2} \widetilde{u}_{2}^{i} \widetilde{\sigma}_{22}^{j-i-1}+\left(\partial_{3} \widetilde{u}_{2}^{i}-\partial_{2} \widetilde{u}_{3}^{i}\right) \widetilde{\sigma}_{23}^{j-i-1}\right)\right. \\
& \left.-\sum_{i=0}^{j} \partial_{2} \widetilde{u}_{1}^{i} \widetilde{\sigma}_{12}^{j-i}\right]+\widetilde{\sigma}_{22}^{j}-\partial_{1}^{2} \widetilde{\sigma}_{22}^{j-1}-\partial_{k}^{2} \widetilde{\sigma}_{22}^{j}=2 r \partial_{2} \widetilde{u}_{2}^{j} \\
& \mathrm{We}\left[\sum_{i=0}^{j-1} \widetilde{u}_{\alpha}^{i} \partial_{\alpha} \widetilde{\sigma}_{23}^{j-i-1}-\frac{1}{2} \sum_{i=0}^{j}\left(\partial_{3} \widetilde{u}_{1}^{i} \widetilde{\sigma}_{12}^{j-i}+\partial_{2} \widetilde{u}_{1}^{i} \widetilde{\sigma}_{13}^{j-i}\right)\right. \\
& \left.+\frac{1}{2} \sum_{i=0}^{j-1}\left(\partial_{1} \widetilde{u}_{3}^{i} \widetilde{\sigma}_{12}^{j-i-1}+\left(\partial_{2} \widetilde{u}_{3}^{i}-\partial_{3} \widetilde{u}_{2}^{i}\right)\left(\widetilde{\sigma}_{22}^{j-i-1}-\widetilde{\sigma}_{33}^{j-i-1}\right)+\partial_{1} \widetilde{u}_{2}^{i} \widetilde{\sigma}_{13}^{j-i-2}\right)\right] \\
& +\widetilde{\sigma}_{23}^{j}-\partial_{1}^{2} \widetilde{\sigma}_{23}^{j-1}-\partial_{k}^{2} \widetilde{\sigma}_{23}^{j}=r\left(\partial_{2} \widetilde{u}_{3}^{j}+\partial_{3} \widetilde{u}_{2}^{j}\right) \\
& \mathrm{We}\left[\sum_{i=0}^{j-1}\left(\widetilde{u}_{\alpha}^{i} \partial_{\alpha} \widetilde{\sigma}_{33}^{j-i-1}+\partial_{1} \widetilde{u}_{3}^{i} \widetilde{\sigma}_{13}^{j-i-1}+\partial_{3} \widetilde{u}_{3}^{i} \widetilde{\sigma}_{33}^{j-i-1}+\left(\partial_{2} \widetilde{u}_{3}^{i}-\partial_{3} \widetilde{u}_{2}^{i}\right) \widetilde{\sigma}_{23}^{j-i-1}\right)\right. \\
& \left.-\sum_{i=0}^{j} \partial_{3} \widetilde{u}_{1}^{i} \widetilde{\sigma}_{13}^{j-i}\right]+\widetilde{\sigma}_{33}^{j}-\partial_{1}^{3} \widetilde{\sigma}_{33}^{j-1}-\partial_{k}^{2} \widetilde{\sigma}_{33}^{j}=2 r \partial_{3} \widetilde{u}_{3}^{j}
\end{aligned}
$$

- The incompressibility relation becomes:

$$
\partial_{1} \widetilde{u}_{1}^{j}+\partial_{k} \widetilde{u}_{k}^{j}=0 .
$$

- The boundary conditions are expected to become (for any $j \geq 0$ again):

$$
\begin{array}{ll}
\left.\widetilde{u}_{\alpha}^{j}\right|_{\Gamma}=0 & \forall \alpha \in\{1,2,3\}, \\
\left.\tilde{\sigma}_{\alpha \beta}^{j}\right|_{\Gamma}=0 & \forall \alpha, \beta \in\{1,2,3\}, \\
\left.\widetilde{u}^{j}\right|_{\Sigma_{0}}=\left.\widetilde{u}^{j}\right|_{\Sigma_{1}},\left.\quad \widetilde{\sigma}^{j}\right|_{\Sigma_{0}}=\left.\widetilde{\sigma}^{j}\right|_{\Sigma_{1}}, & \\
\left.\partial_{1} \widetilde{u}^{j}\right|_{\Sigma_{0}}=\left.\partial_{1} \widetilde{u}^{j}\right|_{\Sigma_{1}}, & \left.\partial_{1} \widetilde{\sigma}^{j}\right|_{\Sigma_{0}}=\left.\partial_{1} \widetilde{\sigma}^{j}\right|_{\Sigma_{1}}, \\
\left.\widetilde{p}^{0}\right|_{\Sigma_{0}}-\left.\widetilde{p}^{0}\right|_{\Sigma_{1}}=p_{d}, & \\
\left.\widetilde{p}^{j}\right|_{\Sigma_{i}}=0 & \forall i \in\{0,1\} \quad \text { (only for } j \geq 1 \text { ). }
\end{array}
$$




\subsection{Main order}

By taking $j=0$ in (V1), (E2), (E3) and $j=-1$ in (V2), (V3) we derive

$$
\left\{\begin{aligned}
-(1-r) \Delta_{k} \widetilde{u}_{1}^{0}+\partial_{1} \widetilde{p}^{0} & =\widetilde{f}_{1}+\partial_{2} \widetilde{\sigma}_{12}^{0}+\partial_{3} \widetilde{\sigma}_{13}^{0}, \\
\widetilde{\sigma}_{12}^{0}-\Delta_{k} \widetilde{\sigma}_{12}^{0} & =r \partial_{2} \widetilde{u}_{1}^{0}, \\
\widetilde{\sigma}_{13}^{0}-\Delta_{k} \widetilde{\sigma}_{13}^{0} & =r \partial_{3} \widetilde{u}_{1}^{0} \\
\partial_{2} \widetilde{p}^{0} & =0 \\
\partial_{3} \widetilde{p}^{0} & =0
\end{aligned}\right.
$$

where $\Delta_{k}=\partial_{2}^{2}+\partial_{3}^{2}$, and the set of equations described in $\left(P_{1}^{0}\right)$ is defined on the domain $\Omega=\left\{\left(x_{1}, x_{2}, x_{3}\right) \in \mathbb{R}^{3} \mid x_{1} \in\right.$ $\left.(0,1),\left(x_{2}, x_{3}\right) \in S\left(x_{1}\right)\right\}$. By taking in $(\mathrm{BC})$ we get

$$
\left\{\begin{array}{rlrl}
\widetilde{u}_{1}^{0}\left(x_{1}, \cdot\right) & =0, & & \text { on } \partial S\left(x_{1}\right), x_{1} \in(0,1), \\
\widetilde{\sigma}_{12}^{0}\left(x_{1}, \cdot\right) & =0, & & \text { on } \partial S\left(x_{1}\right), x_{1} \in(0,1), \\
\widetilde{\sigma}_{13}^{0}\left(x_{1}, \cdot\right) & =0, & & \text { on } \partial S\left(x_{1}\right), x_{1} \in(0,1), \\
\widetilde{u}_{1}^{0} \mid \Sigma_{0} & =\widetilde{u}_{1}^{0} \mid \Sigma_{1}, & & \\
\widetilde{\sigma}_{1 k}^{0} \mid \Sigma_{0} & =\widetilde{\sigma}_{1 k}^{0} \mid \Sigma_{1}, & & \\
\partial_{1} \widetilde{u}_{1}^{0} \mid \Sigma_{0} & = & \partial_{1} \widetilde{u}_{1}^{0} \Sigma_{1}, & \\
\partial_{1} \widetilde{\sigma}_{1 k}^{0} \mid \Sigma_{0} & =\partial_{1} \widetilde{\sigma}_{1 k}^{0} \mid \Sigma_{1}, & & \\
\widetilde{p}^{0}(0)-\widetilde{p}^{0}(1) & =p_{d}, &
\end{array}\right.
$$

We shall use the superposition principle to solve this problem. First, consider - for all $x_{1} \in(0,1)$ - the bi-dimensional problem

$$
\begin{cases}-(1-r) \Delta_{k} \widetilde{v}=\widetilde{f}_{1} & \text { on } S\left(x_{1}\right) \\ \widetilde{v}=0 & \text { on } \partial S\left(x_{1}\right) .\end{cases}
$$

Call $\widetilde{v}_{1}$ the unique smooth solution of this Dirichlet problem (the source term $\widetilde{f}_{1}$ being a scalar, the solution $\widetilde{v}_{1}$ takes values in $\mathbb{R}$ ), and let us denote

$$
\gamma_{1}\left(x_{1}\right)=\int_{S\left(x_{1}\right)} \partial_{1} \widetilde{v}_{1}\left(x_{1}, \cdot\right)
$$

Secondly, consider the problem ( $\widetilde{v}$ being a scalar function - with values in $\mathbb{R}$, and $\widetilde{\tau}$ being a vectorial function - with values in $\mathbb{R}^{2}$ ):

$$
\begin{cases}-(1-r) \Delta_{k} \widetilde{v}+1=\operatorname{div}_{k} \widetilde{\tau} & \text { on } S\left(x_{1}\right), \\ \widetilde{\tau}-\Delta_{k} \widetilde{\tau}=r \nabla_{k} \widetilde{v} & \text { on } S\left(x_{1}\right), \\ \widetilde{v}=0 \quad \text { and } \quad \widetilde{\tau}=0 & \text { on } \partial S\left(x_{1}\right) .\end{cases}
$$

and let us denote

$$
\gamma_{2}\left(x_{1}\right)=\int_{S\left(x_{1}\right)} \partial_{1} \widetilde{v}_{2}\left(x_{1}, \cdot\right) .
$$

Remark 1. Note that the system has a unique solution. The existence part can be proved via a classical approximation technique, using the following energy estimate:

$$
\begin{aligned}
E(\widetilde{v}, \widetilde{\tau}) & :=r(1-r)\left\|\nabla_{k} \widetilde{v}\right\|_{L^{2}\left(S\left(x_{1}\right)\right)}^{2}+\|\widetilde{\tau}\|_{L^{2}\left(S\left(x_{1}\right)\right)}^{2}+\left\|\nabla_{k} \widetilde{\tau}\right\|_{L^{2}\left(S\left(x_{1}\right)\right)}^{2} \\
& =-r \int_{S\left(x_{1}\right)} \widetilde{v} \leq r\left\|\nabla_{k} \widetilde{v}\right\|_{L^{2}\left(S\left(x_{1}\right)\right)} .
\end{aligned}
$$

To show the uniqueness, observe that, if $\left(\widetilde{v}^{(1)}, \widetilde{\tau}^{(1)}\right)$ and $\left(\widetilde{v}^{(2)}, \widetilde{\tau}^{(2)}\right)$ are two solutions then

$$
E\left(\widetilde{v}^{(1)}-\widetilde{v}^{(2)}, \widetilde{\tau}^{(1)}-\widetilde{\tau}^{(2)}\right)=0,
$$

and the conclusion easily follows. The regularity of the solution can be shown by alternating between the two elliptic problems (considered separately) - see [2]. 
Let us call this solution $\left(\widetilde{v}_{2}, \widetilde{\tau}_{2}, \widetilde{\tau}_{3}\right)$. Define

$$
\left(\widetilde{u}_{1}^{0}, \widetilde{\sigma}_{12}^{0}, \widetilde{\sigma}_{13}^{0}\right)=\left(\widetilde{v}_{1}+\partial_{1} \widetilde{p}^{0} \widetilde{v}_{2}, \partial_{1} \widetilde{p}^{0} \widetilde{\tau}_{2}, \partial_{1} \widetilde{p}^{0} \widetilde{\tau}_{3}\right),
$$

with $\widetilde{p}^{0}$ determined by imposing

$$
-\partial_{1}\left(\gamma_{2}\left(x_{1}\right) \partial_{1} \widetilde{p}^{0}\left(x_{1}\right)\right)=\gamma\left(x_{1}\right) \quad \text { and } \quad \widetilde{p}^{0}(0)-\widetilde{p}^{0}(1)=p_{d} .
$$

Note that $\widetilde{p_{0}}$ is defined up to a constant, but $\widetilde{u}_{1}^{0}, \widetilde{\sigma}_{12}^{0}$ and $\widetilde{\sigma}_{13}^{0}$ are uniquely defined. Due to the imposed regularity requirements, we have $\widetilde{u}_{1}^{0}, \widetilde{\sigma}_{12}^{0}, \widetilde{\sigma}_{13}^{0} \in C^{2}(\bar{\Omega})$ and $\widetilde{p}^{0} \in C^{1}(\bar{\Omega})$. It is clear that $\left(\widetilde{u}_{1}^{0}, \widetilde{p}^{0}, \widetilde{\sigma}_{12}^{0}, \widetilde{\sigma}_{13}^{0}\right)$ satisfy $\left(P_{1}^{0}\right)$ in the classical sense. The geometrical assumption on the domain implies that $\widetilde{u}_{1}^{0}\left(x_{1}\right)=\widetilde{u}_{1}^{0}\left(1-x_{1}\right)$ and $\widetilde{\sigma}_{1 k}^{0}\left(x_{1}\right)=\widetilde{\sigma}_{1 k}^{0}\left(1-x_{1}\right)$ for $x_{1} \in[0, \delta)$. Let us call such functions periodic. Clearly all combinations of periodic functions (sum, product, multiplication with scalar) rests periodic. Moreover, as for all periodic functions, $\partial_{1} \widetilde{u}_{1}^{0}(0)=\partial_{1} \widetilde{u}_{1}^{0}(1)=\partial_{1} \widetilde{\sigma}_{1 k}(0)=\partial_{1} \widetilde{\sigma}_{1 k}(1)=0$, so that all conditions in $\left(B C^{0}\right)$ are satisfied. Moreover, the following compatibility condition is verified:

$$
\int_{S\left(x_{1}\right)} \partial_{1} \widetilde{u}_{1}^{0}=0
$$

\subsection{Next orders}

Next, proceed by induction. Let $j \geq 0$. Suppose we have determined smooth periodic $\widetilde{u}_{1}^{i}, \widetilde{\sigma}_{1 k}^{i}, \widetilde{p}^{i}$ with $\int_{S\left(x_{1}\right)} \partial_{1} \widetilde{u}_{1}^{i}=0$ for all $i \leq j$, and $\widetilde{\sigma}_{11}^{i}, \widetilde{u}_{k}^{i}, \widetilde{\sigma}_{k \ell}^{i}$ for all $i<j$ (nothing if $j=0$ ). We show how to determine smooth $\widetilde{\sigma}_{11}^{j}, \widetilde{u}_{k}^{j}, \widetilde{\sigma}_{k \ell}^{j}$ and $\widetilde{u}_{1}^{j+1}, \widetilde{\sigma}_{1 k}^{j+1}$, $\widetilde{p}^{j+1}-$ all periodic - with $\int_{S\left(x_{1}\right)} \partial_{1} \widetilde{u}_{1}^{j+1}=0$.

$\checkmark$ Determination of $\widetilde{\sigma}_{11}^{j}$ : Using (E1) we first derive

$$
\widetilde{\sigma}_{11}^{j}-\partial_{k}^{2} \widetilde{\sigma}_{11}^{j}=\widetilde{f}_{11}^{j}
$$

with known smooth periodic $\widetilde{f}_{11}^{j}$, which can be solved as an elliptic problem - with homogeneous Dirichlet boundary conditions - on every $S\left(x_{1}\right): \tilde{\sigma}_{11}^{j}$ is then known.

$\checkmark$ Determination of $\widetilde{u}_{k}^{j}, \widetilde{\sigma}_{k \ell}^{j}$ and $\widetilde{p}^{j+1}$ : By considering (E4),(E5) and (E6), and also (V2), (V3) and (D) we obtain

$$
\left\{\begin{array}{l}
-(1-r) \partial_{k}^{2} \widetilde{u}_{2}^{j}+\partial_{2} \widetilde{p}^{j+1}=\partial_{k} \widetilde{\sigma}_{2 k}^{j}+\widetilde{g}_{2}^{j}, \\
-(1-r) \partial_{k}^{2} \widetilde{u}_{3}^{j}+\partial_{3} \widetilde{p}^{j+1}=\partial_{k} \widetilde{\sigma}_{3 k}^{j}+\widetilde{g}_{3}^{j}, \\
\widetilde{\sigma}_{22}^{j}-\partial_{k}^{2} \widetilde{\sigma}_{22}^{j}=2 r \partial_{2} \widetilde{u}_{2}^{j}+\widetilde{f}_{22}^{j}, \\
\widetilde{\sigma}_{23}^{j}-\partial_{k}^{2} \widetilde{\sigma}_{23}^{j}=r\left(\partial_{2} \widetilde{u}_{3}^{j}+\partial_{3} \widetilde{u}_{2}^{j}\right)+\widetilde{f}_{23}^{j}, \\
\widetilde{\sigma}_{33}^{j}-\partial_{k}^{2} \widetilde{\sigma}_{33}^{j}=2 r \partial_{3} \widetilde{u}_{3}^{j}+\widetilde{f}_{33}^{j}, \\
\partial_{k} \widetilde{u}_{k}^{j}=-\partial_{1} \widetilde{u}_{1}^{j}
\end{array}\right.
$$

where all the $\widetilde{g}^{j}$ 's and $\widetilde{f}^{j}$ 's are known, smooth and periodic. The above is a coupled inhomogeneous Stokes and elliptic system in 2D, and, if we supplement Dirichlet boundary conditions we get a system like

$$
\begin{cases}-(1-r) \Delta_{k} \widetilde{v}^{j}+\nabla_{k} \widetilde{p}^{j+1}=d i v_{k} \widetilde{\tau}^{j}+\widetilde{g}^{j} & \text { on } S\left(x_{1}\right), \\ \widetilde{\tau}^{j}-\Delta_{k} \widetilde{\tau}^{j}=2 r D_{k} \widetilde{v}^{j}+\widetilde{F}^{j} & \text { on } S\left(x_{1}\right), \\ \operatorname{div} \widetilde{v}^{j}=-\partial_{1} \widetilde{u}_{1}^{j} & \text { on } S\left(x_{1}\right), \\ \widetilde{v}^{j}=0 \quad \text { and } \quad \widetilde{\tau}^{j}=0 & \text { on } \partial S\left(x_{1}\right) .\end{cases}
$$

Owing to the condition $\int_{S\left(x_{1}\right)} \partial_{1} \widetilde{u}_{1}^{j}=0$, use a classical result [4] to find $\widetilde{z}^{j}$ (not necessarily smooth) such that

$$
\begin{cases}\operatorname{div}_{k} \widetilde{z}^{j}=-\partial_{1} \widetilde{u}_{1}^{j} & \text { on } S\left(x_{1}\right), \\ \widetilde{z}^{j}=0 & \text { on } \partial S\left(x_{1}\right) .\end{cases}
$$


Evidently we can choose $\widetilde{z}^{j}$ to be periodic. Therefore, in order to prove the existence of a solution to problem $\left(P_{k}^{j}\right)$ it suffices to prove the existence to the following problem

$$
\begin{cases}-(1-r) \Delta_{k} \widetilde{w}^{j}+\nabla_{k} \widetilde{p}^{j+1}=\operatorname{div}_{k} \widetilde{\tau}^{j}+\widetilde{g}^{j, 1} & \text { on } S\left(x_{1}\right), \\ \widetilde{\tau}^{j}-\Delta_{k} \widetilde{\tau}^{j}=2 r D_{k} \widetilde{w}^{j}+\widetilde{F}^{j, 1} & \text { on } S\left(x_{1}\right), \\ \operatorname{div} \widetilde{w}_{k}=0 & \text { on } S\left(x_{1}\right), \\ \widetilde{w}^{j}, \widetilde{\tau}^{j}=0 & \text { on } \partial S\left(x_{1}\right) .\end{cases}
$$

The above system is a linearized version of our initial problem, and the existence of a unique solution is shown using the Lax-Milgram theorem. The regularity is obtained by alternating between the Stokes problem and the elliptic ones in $\left(P_{k}^{j}\right)$. Moreover, since the source terms are periodic, this solution is also periodic. Note that $\widetilde{p}^{j+1}$ is defined up to a constant for all $x_{1}-$ say $\widetilde{r}_{j+1}\left(x_{1}\right)$.

$\checkmark$ Determination of $\widetilde{u}_{1}^{j+1}$ and $\widetilde{\sigma}_{1 k}^{j+1}$ : By taking $j+1$ instead of $j$ in (V1),(E2),(E3) we derive

$$
\left\{\begin{array}{l}
-(1-r) \partial_{k}^{2} \widetilde{u}_{1}^{j+1}+\partial_{1} \widetilde{r}_{j+1}=\widetilde{g}_{1}^{j+1}+\partial_{2} \widetilde{\sigma}_{12}^{j+1}+\partial_{3} \widetilde{\sigma}_{13}^{j+1}, \\
\widetilde{\sigma}_{12}^{j+1}-\partial_{k}^{2} \widetilde{\sigma}_{12}^{j+1}=r \partial_{2} \widetilde{u}_{1}^{j+1}+\widetilde{f}_{12}^{j+1} \\
\widetilde{\sigma}_{13}^{j+1}-\partial_{k}^{2} \widetilde{\sigma}_{13}^{j+1}=r \partial_{3} \widetilde{u}_{1}^{j+1}+\widetilde{f}_{13}^{j+1}
\end{array}\right.
$$

with known, smooth, periodic $\widetilde{g}_{1}^{j+1}, \widetilde{f}_{12}^{j+1}, \widetilde{f}_{13}^{j+1}$. This is handled in exactly the same manner as problem $\left(P_{1}^{0}\right)$, with the sole difference that $\widetilde{r}_{j+1}$ is uniquely defined by the conditions $\widetilde{r}_{j+1}(0)=\widetilde{r}_{j+1}(1)=0$.

\section{$5 \quad$ Error estimates}

Let

$$
\begin{aligned}
& u_{1}^{n}\left(x_{1}, x_{k}\right)=\sum_{j=0}^{n} \varepsilon^{2 j+2} \widetilde{u}_{1}^{j}\left(x_{1}, \frac{x_{k}}{\varepsilon}\right), \\
& u_{\ell}^{n}\left(x_{1}, x_{k}\right)=\sum_{j=0}^{n} \varepsilon^{2 j+3} \widetilde{u}_{k}^{j}\left(x_{1}, \frac{x_{k}}{\varepsilon}\right), \quad \ell \in\{2,3\}, \\
& p^{n}\left(x_{1}, x_{k}\right)=\sum_{j=0}^{n} \varepsilon^{2 j} \widetilde{p}^{j}\left(x_{1}, \frac{x_{k}}{\varepsilon}\right), \\
& \sigma_{11}^{n}\left(x_{1}, x_{k}\right)=\sum_{j=0}^{n} \varepsilon^{2 j+2} \widetilde{\sigma}_{11}^{j}\left(x_{1}, \frac{x_{k}}{\varepsilon}\right), \\
& \sigma_{1 \ell}^{n}\left(x_{1}, x_{k}\right)=\sum_{j=0}^{n} \varepsilon^{2 j+1} \widetilde{\sigma}_{1 \ell}^{j}\left(x_{1}, \frac{x_{k}}{\varepsilon}\right), \quad \ell \in\{2,3\}, \\
& \sigma_{\ell m}^{n}\left(x_{1}, x_{k}\right)=\sum_{j=0}^{n} \varepsilon^{2 j+2} \widetilde{\sigma}_{\ell m}^{j}\left(x_{1}, \frac{x_{k}}{\varepsilon}\right), \quad \ell, m \in\{2,3\} .
\end{aligned}
$$

Proposition 3. Provided $\varepsilon$ is small enough, for all $n \geq 0$, the following estimates hold true:

$$
\begin{aligned}
& \left\|\nabla\left(u-u^{n}\right)\right\| \leq C \varepsilon^{2 n+4}, \\
& \left\|\nabla\left(\sigma-\sigma^{n}\right)\right\| \leq C \varepsilon^{2 n+3}, \\
& \left\|p-p^{n}\right\| \leq C \varepsilon^{2 n+2} .
\end{aligned}
$$

Proof. From the previous section we get that $\left(u^{n}, \sigma^{n}\right) \in V \times W$. Also, $\left(u^{n}, \sigma^{n}, p^{n}\right)$ are smooth and satisfy (2). After some calculations we get

$$
\left\{\begin{array}{l}
-(1-r) \Delta u^{n}+\operatorname{Re}\left(u^{n} \cdot \nabla\right) \cdot u^{n}+\nabla p^{n+1}=\operatorname{div} \sigma^{n}+f_{\varepsilon}+R^{n} \\
\operatorname{We}\left(\left(u^{n} \cdot \nabla\right) \sigma^{n}+g_{0}\left(\sigma^{n}, \nabla u^{n}\right)\right)+\sigma^{n}-\varepsilon^{2} \Delta \sigma^{n}=2 r D u^{n}+Q^{n} \\
\operatorname{div} u^{n}=0
\end{array}\right.
$$


where $R^{n}, Q^{n}$ have complicated expressions that we'd rather omit. Still, as in [6] we can prove

$$
\left\|R^{n}\right\|_{L^{\infty}} \leq C \varepsilon^{2 n+2},\left\|Q^{n}\right\|_{L^{\infty}} \leq C \varepsilon^{2 n+3} .
$$

It easily follows

$$
\left\{\begin{aligned}
(1-r) \int_{\Omega_{\varepsilon}} \nabla u^{n}: \nabla v+\operatorname{Re} \int_{\Omega_{\varepsilon}}\left(u^{n} \cdot \nabla\right) u^{n} \cdot v+\int_{\Omega_{\varepsilon}} \sigma^{n}: D v & \\
= & \int_{\Omega_{\varepsilon}} f_{\varepsilon} v+p_{d} \int_{\Sigma_{\varepsilon}^{0}} v_{1}+\int_{\Omega_{\varepsilon}} R^{n} v, \\
\operatorname{We} \int_{\Omega_{\varepsilon}}\left(\left(u^{n} \cdot \nabla\right) \sigma^{n}+g_{0}\left(\sigma^{n}, \nabla u^{n}\right)\right) & : \tau+\int_{\Omega_{\varepsilon}} \sigma^{n}: \tau+\varepsilon^{2} \int_{\Omega_{\varepsilon}} \nabla \sigma^{n}: \nabla \tau \\
& =2 r \int_{\Omega_{\varepsilon}} D u^{n}: \tau+\int_{\Omega_{\varepsilon}} Q^{n}: \tau .
\end{aligned}\right.
$$

By taking in (3) and (11) $v=u-u^{n}$ and $\tau=\sigma-\sigma^{n}$ and making some elementary manipulations, we obtain

$$
\begin{aligned}
& 2 r(1-r)\left\|\nabla\left(u-u^{n}\right)\right\|^{2}+\left\|\sigma-\sigma^{n}\right\|^{2}+\varepsilon^{2}\left\|\nabla\left(\sigma-\sigma^{n}\right)\right\|^{2} \\
& \leq 2 r\left|\int_{\Omega_{\varepsilon}} R^{n}\left(u-u^{n}\right)\right|+\left|\int_{\Omega_{\varepsilon}} Q^{n}\left(\sigma-\sigma^{n}\right)\right| \\
& \quad+2 r \operatorname{Re}\left(\left|\int_{\Omega_{\varepsilon}}\left(u-u^{n}\right) \cdot \nabla u^{n} \cdot\left(u-u^{n}\right)\right|+\left|\int_{\Omega_{\varepsilon}} u \cdot \nabla\left(u-u^{n}\right) \cdot\left(u-u^{n}\right)\right|\right) \\
& \quad+\operatorname{We}\left(\left|\int_{\Omega_{\varepsilon}}\left(u-u^{n}\right) \cdot \nabla \sigma^{n}:\left(\sigma-\sigma^{n}\right)\right|+\left|\int_{\Omega_{\varepsilon}} u \cdot \nabla\left(\sigma-\sigma^{n}\right):\left(\sigma-\sigma^{n}\right)\right|\right. \\
& \left.\quad+\left|\int_{\Omega_{\varepsilon}} g_{0}\left(\nabla\left(u-u^{n}\right), \sigma^{n}\right):\left(\sigma-\sigma^{n}\right)\right|+\left|\int_{\Omega_{\varepsilon}} g_{0}\left(\nabla u, \sigma-\sigma^{n}\right):\left(\sigma-\sigma^{n}\right)\right|\right) .
\end{aligned}
$$

By using Hölder's inequality, (10), Lemma 1, estimates in Proposition 1, as well as the following trivial $\left\|\nabla \sigma_{n}\right\| \leq C \varepsilon$, we derive the following estimates:

$$
\begin{aligned}
& \left|\int_{\Omega_{\varepsilon}} R^{n} \cdot\left(u-u^{n}\right)\right| \leq C \varepsilon^{2 n+4}\left\|\nabla\left(u^{n}-u\right)\right\|, \\
& \left|\int_{\Omega_{\varepsilon}} Q^{n}\left(\sigma-\sigma^{n}\right)\right| \leq C \varepsilon^{2 n+5}\left\|\nabla\left(\sigma-\sigma^{n}\right)\right\|, \\
& \left|\int_{\Omega_{\varepsilon}}\left(u-u^{n}\right) \cdot \nabla u^{n} \cdot\left(u-u^{n}\right)\right| \leq C \varepsilon^{3}\left\|\nabla\left(u-u^{n}\right)\right\|^{2}, \\
& \left|\int_{\Omega_{\varepsilon}} u \cdot \nabla\left(u-u^{n}\right) \cdot\left(u-u^{n}\right)\right| \leq C \varepsilon^{5 / 2}\left\|\nabla\left(u-u^{n}\right)\right\|^{2}, \\
& \left|\int_{\Omega_{\varepsilon}}\left(u-u^{n}\right) \cdot \nabla \sigma^{n}:\left(\sigma-\sigma^{n}\right)\right| \leq C \varepsilon^{3 / 2}\left\|\nabla\left(u^{n}-u\right)\right\|\left\|\nabla\left(\sigma-\sigma^{n}\right)\right\| \leq C \varepsilon^{1 / 2}\left(\left\|\nabla\left(u^{n}-u\right)\right\|^{2}+\varepsilon^{2}\left\|\sigma-\sigma^{n}\right\|^{2}\right), \\
& \left|\int_{\Omega_{\varepsilon}} u \cdot \nabla\left(\sigma-\sigma^{n}\right):\left(\sigma-\sigma^{n}\right)\right| \leq C \varepsilon^{5 / 2}\left\|\nabla\left(\sigma-\sigma^{n}\right)\right\|^{2}, \\
& \left|\int_{\Omega_{\varepsilon}} g_{0}\left(\nabla\left(u-u^{n}\right), \sigma^{n}\right):\left(\sigma-\sigma^{n}\right)\right| \leq C \varepsilon^{3 / 2}\left\|\nabla\left(u^{n}-u\right)\right\|\left\|\nabla\left(\sigma-\sigma^{n}\right)\right\| \leq C \varepsilon^{1 / 2}\left(\left\|\nabla\left(u^{n}-u\right)\right\|^{2}+\varepsilon^{2}\left\|\sigma-\sigma^{n}\right\|^{2}\right), \\
& \left|\int_{\Omega_{\varepsilon}} g_{0}\left(\nabla u, \sigma-\sigma^{n}\right):\left(\sigma-\sigma^{n}\right)\right| \leq C \varepsilon^{5 / 2}\left\|\nabla\left(\sigma-\sigma^{n}\right)\right\|^{2} .
\end{aligned}
$$

Hence, for $\varepsilon$ small, enough we can derive

$$
\left\|\nabla\left(u-u^{n}\right)\right\|^{2}+\varepsilon^{2}\left\|\nabla\left(\sigma-\sigma^{n}\right)\right\|^{2} \leq C \varepsilon^{2 n+4}\left(\left\|\nabla\left(u-u^{n}\right)\right\|+\left\|\nabla\left(\sigma-\sigma^{n}\right)\right\|\right)
$$


from which follows easily

$$
\begin{aligned}
& \left\|\nabla\left(u-u^{n}\right)\right\| \leq C \varepsilon^{2 n+4} \\
& \left\|\nabla\left(\sigma-\sigma^{n}\right)\right\| \leq C \varepsilon^{2 n+3}
\end{aligned}
$$

To derive a pressure estimate, use the result in [6] to find $\phi_{n} \in\left(H_{0}^{1}\left(\Omega_{\varepsilon}\right)\right)^{3}$ such that:

$$
\begin{aligned}
& \operatorname{div} \phi_{n}=p-p^{n+1} \quad \text { on } \quad \Omega_{\varepsilon}, \\
& \left\|\nabla \phi_{n}\right\| \leq C \varepsilon^{-1}\left\|p-p^{n+1}\right\| .
\end{aligned}
$$

Consider the first equation in (1) - understood in distributional sense - and (9). By subtracting the two and then applying $\phi_{n} \in\left(H_{0}^{1}\left(\Omega_{\varepsilon}\right)\right)^{3}$ we obtain

$$
\begin{aligned}
\left\|p-p^{n+1}\right\|^{2} & +(1-r) \int_{\Omega_{\varepsilon}} \nabla\left(u-u^{n}\right) \cdot \nabla \phi_{n} \\
& =\int_{\Omega_{\varepsilon}}\left(\left(u^{n} \cdot \nabla\right) u^{n}-(u \cdot \nabla) u\right) \cdot \phi_{n}-\int_{\Omega_{\varepsilon}} R^{n} \cdot \phi_{n} .
\end{aligned}
$$

Proceeding in a very similar manner to the way we determined the velocity estimates - while also using these estimates we establish

$$
\left\|p-p^{n+1}\right\| \leq C \varepsilon^{2 n+3},
$$

from which the pressure estimate easily follows.

Remark 2. By replicating the proof of Proposition (3), we can prove that, for $\varepsilon$ small enough, the solution to problem (1)-(2) is unique.

\section{Axisymmetric equation}

In view of the last remark in the previous section, we shall suppose that $\varepsilon$ is small enough so that the solution to our problem is uniquely defined.

Henceforth, suppose that the domain $\Omega_{\varepsilon}$ is axisymmetric, namely that is obtained by rotating around the $x_{1}$ axis the following $2 \mathrm{D}$ domain

$$
D_{\varepsilon}=\left\{\left(x_{1}, x_{3}\right) \in \mathbb{R}^{2} \mid x_{1} \in(0,1), \quad x_{3} \in\left(0, \varepsilon h\left(x_{1}\right)\right)\right\} .
$$

for some smooth $h:[0,1] \rightarrow \mathbb{R}_{+}^{*}$, such that $h\left(x_{1}\right)=h(0)=h(1)=h\left(1-x_{1}\right)$ for all $x_{1} \in(0, \delta)$.

A comprehensive study of various equations in axisymmetric domains can be found in [1]. The goal of this section is to show that the weak solution is "axisymmetric" in a sense that we shall properly define provided the source term enjoys the same property. In [8] a characterization of regular axisymmetric functions related to the Navier-Stokes equation is given. Here we show how to extend those results to the case of weak solutions. Although the result is very intuitive, the proof - while elementary - is quite technical. We start by introducing cylindrical coordinates

$$
\begin{aligned}
& x_{1}=x, \\
& x_{2}=z \sin \theta, \\
& x_{3}=z \cos \theta,
\end{aligned}
$$

with $x \in[0,1], z \in[0, \varepsilon h(x)], \theta \in[0,2 \pi)$. We write

$$
\left\{\begin{array}{l}
u=u_{x}(x, z, \theta) e_{x}+u_{z}(x, z, \theta) e_{z}+u_{\theta}(x, z, \theta) e_{\theta} \\
\sigma=\sigma_{x x}(x, z, \theta) e_{x} \otimes e_{x}+\cdots+\sigma_{\theta \theta}(x, z, \theta) e_{\theta} \otimes e_{\theta}
\end{array}\right.
$$

with

$$
e_{x}=\left(\begin{array}{l}
1 \\
0 \\
0
\end{array}\right), \quad e_{z}=\left(\begin{array}{c}
0 \\
\sin \theta \\
\cos \theta
\end{array}\right), \quad e_{\theta}=\left(\begin{array}{c}
0 \\
\cos \theta \\
-\sin \theta
\end{array}\right) .
$$


Consider

$$
\mathcal{X}=C^{1}\left(\Omega_{\varepsilon}\right) \cap C\left(\overline{\Omega_{\varepsilon}}\right) \cap H^{1}\left(\Omega_{\varepsilon}\right) .
$$

Note that if $u \in \mathcal{X}^{3}$ and $\sigma \in \mathbb{M}_{3 \times 3}(\mathcal{X})$ then $u_{x}, u_{z}, u_{\theta}, \sigma_{x x}, \ldots, \sigma_{\theta \theta} \in C^{1}\left(D_{\varepsilon} \times(0,2 \pi)\right)$, and so we can define

$$
\begin{aligned}
& \mathcal{X}_{s}=\left\{u \in \mathcal{X}^{3} \mid \partial_{\theta} u_{x}=\partial_{\theta} u_{z}=\partial_{\theta} u_{\theta}=0\right\}, \\
& \mathcal{Y}_{s}=\left\{\sigma \in \mathbb{M}_{3 \times 3}(\mathcal{X}) \mid \sigma_{i j}=\sigma_{j i}, \quad \partial_{\theta} \sigma_{x x}=\cdots=\partial_{\theta} \sigma_{\theta \theta}=0\right\} .
\end{aligned}
$$

Lemma 3. Given $u \in \mathcal{X}^{3}$ then $u_{x}, u_{z}, u_{\theta} \in C\left(\overline{D_{\varepsilon} \times(0,2 \pi)}\right)$, and for all $\theta \in(0,2 \pi]$ we have $u_{x}(\cdot, \cdot, \theta), u_{z}(\cdot, \cdot, \theta), u_{\theta}(\cdot, \cdot, \theta) \in$ $C^{1}\left(D_{\varepsilon}^{\prime}\right)$ where $D_{\varepsilon}^{\prime}=D_{\varepsilon} \cup\{(x, 0) \mid x \in(0,1)\}$. Moreover, for all $x \in(0,1)$ and $\theta \in[0, \pi)$ we have

$$
\begin{aligned}
& u_{z}(x, 0, \theta)=-u_{z}(x, 0, \theta+\pi), \\
& u_{\theta}(x, 0, \theta)=-u_{\theta}(x, 0, \theta+\pi), \\
& \partial_{z} u_{x}(x, 0, \theta)=-\partial_{z} u_{x}(x, 0, \theta+\pi) .
\end{aligned}
$$

In particular, if $u \in \mathcal{X}_{s}$ then $u_{z}(x, 0)=u_{\theta}(x, 0)=0$ and $\partial_{z} u_{x}(x, 0)=0$.

Proof. The proof is trivial and follows from the definition of $u_{x}, u_{z}, u_{\theta}$.

Observe that if $u \in \mathcal{X}^{3}$ then

$$
\int_{\partial \Omega_{\varepsilon}} u^{2}=\int_{T_{\varepsilon}}\left(u_{x}^{2}+u_{z}^{2}+u_{\theta}^{2}\right) z
$$

where $T_{\varepsilon}=\partial\left(D_{\varepsilon} \times(0,2 \pi)\right) \backslash\{(x, 0, \theta) \mid x \in(0,1), \theta \in(0,2 \pi)\}$. Also, if $u \in \mathcal{X}_{s}$ then

$$
\begin{aligned}
\|u\|_{H^{1}}^{2} & =2 \pi \int_{D_{\varepsilon}}\left(u_{x}^{2}+u_{z}^{2}+u_{\theta}^{2}\right) z+\frac{u_{z}^{2}}{z}+\frac{u_{\theta}^{2}}{z} \\
& +2 \pi \int_{D_{\varepsilon}}\left(\left(\partial_{x} u_{x}\right)^{2}+\left(\partial_{z} u_{x}\right)^{2}+\left(\partial_{x} u_{z}\right)^{2}+\left(\partial_{z} u_{z}\right)^{2}+\left(\partial_{x} u_{\theta}\right)^{2}+\left(\partial_{z} u_{\theta}\right)^{2}\right) z .
\end{aligned}
$$

This motivates the introduction of the following spaces

$$
\begin{aligned}
& H=\left\{u \in L_{z}^{2}\left(D_{\varepsilon}\right) \mid \nabla u \in\left(L_{z}^{2}\left(D_{\varepsilon}\right)\right)^{2}\right\} \\
& H^{\prime}=\left\{u \in L_{z^{-1}}^{2}\left(D_{\varepsilon}\right) \mid \nabla u \in\left(L_{z}^{2}\left(D_{\varepsilon}\right)\right)^{2}\right\}
\end{aligned}
$$

where, if $w \geq 0$ on $D_{\varepsilon}$, then $L_{w}^{2}\left(D_{\varepsilon}\right)$ is the classical weighted Lebesgue space, endowed with the scalar product

$$
(u, v)_{L_{w}^{2}}=\int_{D_{\varepsilon}} u v w .
$$

The spaces $H$ and $H^{\prime}$ become Hilbert spaces when endowed with the following scalar products:

$$
\begin{aligned}
& (u, v)_{H}=2 \pi\left((u, v)_{L_{z}^{2}}+\left(\partial_{x} u, \partial_{x} v\right)_{L_{z}^{2}}+\left(\partial_{z} u, \partial_{z} v\right)_{L_{z}^{2}}\right) \\
& (u, v)_{H^{\prime}}=2 \pi\left((u, v)_{L_{z}^{2}}+(u, v)_{L_{z^{-1}}^{2}}+\left(\partial_{x} u, \partial_{x} v\right)_{L_{z}^{2}}+\left(\partial_{z} u, \partial_{z} v\right)_{L_{z}^{2}}\right)
\end{aligned}
$$

The application $T:\left(\mathcal{X}_{s},\|\cdot\|_{H^{1}}\right) \rightarrow H \times H^{\prime} \times H^{\prime}$ defined by $T u=\left(u_{x}, u_{z}, u_{\theta}\right)$ is an isometry, and so if $X_{s}$ is the completion of $\mathcal{X}_{s}$ in $H^{1}$ then $T$ can be uniquely extended to an isometry - still denoted by $T: X_{s} \rightarrow H \times H^{\prime} \times H^{\prime}$.

As we could see in Lemma 3 , if $u \in \mathcal{X}_{s}$ then $u_{x}, u_{z}, u_{\theta} \in \mathcal{C}=\left\{\left(v_{x}, v_{z}, v_{\theta}\right) \in C^{1}\left(D_{\varepsilon}^{\prime}\right) \cap C\left(\overline{D_{\varepsilon}}\right) \mid \partial_{z} v_{x}(x, 0)=0, v_{z}(x, 0)=\right.$ $\left.v_{\theta}(x, 0)=0\right\}$. We can now show that this completely defines the elements in $\mathcal{X}_{s}$.

Lemma 4. The operator $T$ is bijective from $\mathcal{X}_{s}$ to $\mathcal{C}$.

Proof. Given $\left(u_{x}, u_{z}, u_{\theta}\right) \in \mathcal{C}$, then we have

$$
\begin{aligned}
& u_{1}\left(x_{1}, x_{2}, x_{3}\right)=u_{x}\left(x_{1}, z\right), \\
& u_{2}\left(x_{1}, x_{2}, x_{3}\right)=u_{z}\left(x_{1}, z\right) \frac{x_{2}}{z}+u_{\theta}\left(x_{1}, z\right) \frac{x_{3}}{z}, \\
& u_{3}\left(x_{1}, x_{2}, x_{3}\right)=u_{z}\left(x_{1}, z\right) \frac{x_{3}}{z}-u_{\theta}\left(x_{1}, z\right) \frac{x_{2}}{z},
\end{aligned}
$$


with $r=\sqrt{x_{2}^{2}+x_{3}^{2}}$. The only non-trivial part is to show that $u, \nabla u$ are well-defined on $z=0$. This is obvious for $u_{1}$ and $\partial_{1} u_{1}$, while

$$
\partial_{k} u_{1}\left(x_{1}, x_{2}, x_{3}\right)=\partial_{z} u_{x}\left(x_{1}, z\right) \frac{x_{k}}{z}
$$

(for $k \in\{2,3\}$ ) is 0 on $z=0$, as the product of a function which tends to 0 as $z \rightarrow 0$ and a bounded function. The same idea is used to show that $u_{k}, \partial_{1} u_{k}$ are 0 on $z=0$, for $k \in\{2,3\}$. Finally,

$$
\begin{gathered}
\partial_{2} u_{2}\left(x_{1}, x_{2}, x_{3}\right)=\partial_{z} u_{z}\left(x_{1}, z\right) \frac{x_{2}^{2}}{z^{2}}+u_{z}\left(x_{1}, z\right) \frac{x_{3}^{2}}{z^{3}}+\partial_{z} u_{\theta}\left(x_{1}, z\right) \frac{x_{2} x_{3}}{z^{2}}-u_{\theta}\left(x_{1}, z\right) \frac{x_{2} x_{3}}{z^{3}} \\
=\partial_{z} u_{z}\left(x_{1}, z\right)+\left(\frac{u_{z}\left(x_{1}, z\right)}{z}-\partial_{z} u_{z}\left(x_{1}, z\right)\right) \frac{x_{3}^{2}}{z^{2}}+\frac{x_{2} x_{3}}{z^{2}}\left(\frac{-u_{\theta}\left(x_{1}, z\right)}{z}+\partial_{z} u_{\theta}\left(x_{1}, z\right)\right) .
\end{gathered}
$$

Since $u_{z}\left(x_{1}, 0\right)=u_{\theta}\left(x_{1}, 0\right)=0$, the second and the third term in the above equation are convergent to 0 , while the first is convergent to $\partial_{z} u_{z}\left(x_{1}, 0\right)$. The rest of the terms are treated exactly in the same manner.

Clearly, all the above considerations can be extended to $\sigma$, and we define $Y_{s}$ to be the completion of $\mathcal{Y}_{s}$ with respect to the $H^{1}$ norm. Define

$$
\begin{aligned}
& V_{s}=\left\{u \in X_{s} \cap\left(H_{\#}^{1}\right)^{3} \mid \text { div } u=0, \quad u=0 \text { on } \Gamma_{\varepsilon}\right\} \\
& W_{s}=\left\{\sigma \in Y_{s} \cap \mathbb{M}_{3 \times 3}\left(H_{\#}^{1}\right) \mid \sigma=0 \text { on } \Gamma_{\varepsilon}\right\} .
\end{aligned}
$$

Let us denote $L_{1}(u, \sigma)$ and $L_{2}(u, \sigma)$ the operators involved in the variational formulation (3). We have proven in the first part that there exists exactly one solution to the problem

$$
\begin{cases}\left\langle L_{1}(u, \sigma), v\right\rangle=0 & \text { for all } v \in V, \\ \left\langle L_{2}(u, \sigma), \tau\right\rangle=0 & \text { for all } \tau \in W .\end{cases}
$$

In a completely similar manner, it can be proven that there is $(\widehat{u}, \widehat{\sigma}) \in V_{s} \times W_{s}$ such that

$$
\begin{cases}\left\langle L_{1}(\widehat{u}, \widehat{\sigma}), \widehat{v}\right\rangle=0 & \text { for all } \widehat{v} \in V_{s}, \\ \left\langle L_{2}(\widehat{u}, \widehat{\sigma}), \widehat{\tau}\right\rangle=0 & \text { for all } \widehat{\tau} \in W_{s} .\end{cases}
$$

We intend to prove that $(\widehat{u}, \widehat{\sigma})$ is in fact a solution to $(3)$. The key ingredient will be the following:

Lemma 5. Assume that $f \in \mathcal{X}_{s}$. Then there exists bounded operators $\Phi: V \rightarrow V_{s}$ and $\Psi: W \rightarrow W_{s}$ with $\Phi v:=v^{s}$ and $\Psi \tau:=\tau^{s}$ such that, for all $(v, \tau) \in V \times W$,

$$
\begin{aligned}
& \left\langle L_{1}(\widehat{u}, \widehat{\sigma}), v\right\rangle=\left\langle L_{1}(\widehat{u}, \widehat{\sigma}), v^{s}\right\rangle, \\
& \left\langle L_{2}(\widehat{u}, \widehat{\sigma}), \tau\right\rangle=\left\langle L_{2}(\widehat{u}, \widehat{\sigma}), \tau^{s}\right\rangle .
\end{aligned}
$$

Note that this readily implies that $(\widehat{u}, \widehat{\sigma})$ is solution to the variational problem $(3)$.

Proof. We prove only for $\Phi$, since the other one is similar. Let $v \in \mathcal{X}^{3}$. Then, by Lemma 3 we have $v_{x}, v_{z}, v_{\theta} \in$ $C\left(\overline{D_{\varepsilon} \times(0,2 \pi)}\right)$, and for all $\theta \in[0,2 \pi)$ we have $v_{x}(\cdot, \cdot, \theta), v_{z}(\cdot, \cdot, \theta), v_{\theta}(\cdot, \cdot, \theta) \in C^{1}\left(D_{\varepsilon}^{\prime}\right)$. Denote

$$
\begin{aligned}
& v_{x}^{s}(x, z)=\frac{1}{2 \pi} \int_{0}^{2 \pi} v_{x}(x, z, \theta) d \theta, \\
& v_{z}^{s}(x, z)=\frac{1}{2 \pi} \int_{0}^{2 \pi} v_{z}(x, z, \theta) d \theta, \\
& v_{\theta}^{s}(x, z)=\frac{1}{2 \pi} \int_{0}^{2 \pi} v_{\theta}(x, z, \theta) d \theta .
\end{aligned}
$$

Clearly $v_{x}^{s}, v_{z}^{s}, v_{\theta}^{s} \in C\left(\overline{D_{\varepsilon}}\right) \cap C^{1}\left(D_{\varepsilon}^{\prime}\right)$, and from (12a), (12b), (12c) it follows that $\partial_{z} v_{x}^{s}(x, 0)=0, v_{z}^{s}(x, 0)=v_{\theta}^{s}(x, 0)=0$. Hence, from Lemma 4, we get that $v^{s}\left(=T^{-1}\left(v_{x}^{s}, v_{z}^{s}, v_{\theta}^{s}\right)\right) \in \mathcal{X}_{s}$. Moreover, it is elementary to see that

$$
\left\|v^{s}\right\|_{H^{1}\left(\Omega_{\varepsilon}\right)} \leq\|v\|_{H^{1}\left(\Omega_{\varepsilon}\right)}
$$


If we denote $v^{a s}=v-v^{s}$, then we have the following

$$
\int_{0}^{2 \pi} v_{x}^{a s}(x, z, \theta) d \theta=\int_{0}^{2 \pi} v_{z}^{a s}(x, z, \theta) d \theta=\int_{0}^{2 \pi} v_{\theta}^{a s}(x, z, \theta) d \theta=0 .
$$

So if $(u, \sigma) \in \mathcal{X}_{s} \times \mathcal{Y}_{s}$, then by a change of variables it can be proved that

$$
\left\langle L_{1}(u, \sigma), v^{a s}\right\rangle=0
$$

and so by a density argument it follows

$$
\left\langle L_{1}(\widehat{u}, \widehat{\sigma}), v\right\rangle=\left\langle L_{1}(\widehat{u}, \widehat{\sigma}), v^{s}\right\rangle,
$$

which implies that (13a) is true for all $v \in \mathcal{X}^{3}$. From (14) we get that $\Phi$ is continuous on $\mathcal{X}^{3}$, and so it can be uniquely extended to $H^{1}$, and (14) is still verified for all $v \in H^{1}$. To conclude we need to show that $\Phi(V) \subset V_{s}$, that is, for all $v \in V, v^{s}$ is divergence free and satisfies the same boundary conditions as $v$. The continuity of $\Phi$ readily implies that $v^{s}=0$ on $\Gamma_{\varepsilon}$, while using the following inequalities

$$
\begin{array}{ll}
\left\|\operatorname{div} v^{s}\right\|_{L^{2}\left(\Omega_{\varepsilon}\right)} \leq\|\operatorname{div} v\|_{L^{2}\left(\Omega_{\varepsilon}\right)} & \forall v \in \mathcal{X}^{3} \\
\left\|v^{s}(0)-v^{s}(1)\right\|_{L^{2}\left(\Sigma_{0}^{\varepsilon}\right)} \leq\|v(0)-v(1)\|_{L^{2}\left(\Sigma_{0}^{\varepsilon}\right)} & \forall v \in \mathcal{X}^{3}
\end{array}
$$

which are easily derived by changing of variables, that using Cauchy-Schwarz inequality, we derive that div $v^{s}=0$ and $v^{s} \in H_{\#}^{1}$ through a density argument.

We can actually prove more. Note that

$$
V_{s}=V_{s}^{\prime} \oplus V_{s}^{\prime \prime} \quad \text { and } \quad W_{s}=W_{s}^{\prime} \oplus W_{s}^{\prime \prime},
$$

where

$$
\begin{aligned}
& V_{s}^{\prime}=\left\{v \in V_{s} \mid v_{\theta}=0\right\}, \\
& V_{s}^{\prime \prime}=\left\{v \in V_{s} \mid v_{x}=v_{z}=0\right\}, \\
& W_{s}^{\prime}=\left\{\sigma \in W_{s} \mid \sigma_{x \theta}=\sigma_{z \theta}=0\right\}, \\
& W_{s}^{\prime \prime}=\left\{\sigma \in W_{s} \mid \sigma_{x x}=\sigma_{x z}=\sigma_{z z}=\sigma_{\theta \theta}=0\right\} .
\end{aligned}
$$

We intend to show that, provided $f \in\left\{g \in \mathcal{X}_{s} \mid g_{\theta}=0\right\}$, the solution is in $V_{s}^{\prime} \times W_{s}^{\prime}$. We use the same trick as before. First, there exists $(\widetilde{u}, \widetilde{\sigma}) \in V_{s}^{\prime} \times W_{s}^{\prime}$ such that

$$
\begin{aligned}
& \left\langle L_{1}(\widetilde{u}, \widetilde{\sigma}), \widetilde{v}\right\rangle=0, \\
& \left\langle L_{2}(\widetilde{u}, \widetilde{\sigma}), \widetilde{\tau}\right\rangle=0 .
\end{aligned}
$$

for all $(\widetilde{v}, \widetilde{\tau}) \in V_{s}^{\prime} \times W_{s}^{\prime}$. Pick any $(\widehat{v}, \widehat{\tau}) \in V_{s} \times W_{s}$. Then it can be expressed as $(\widetilde{v}, \widetilde{\tau})+(\bar{v}, \bar{\tau})$ with $(\widetilde{v}, \widetilde{\tau}) \in V_{s}^{\prime} \times W_{s}^{\prime}$ and $(\bar{v}, \bar{\tau}) \in V_{s}^{\prime \prime} \times W_{s}^{\prime \prime}$. To conclude, it rests to prove that

$$
\begin{aligned}
& \left\langle L_{1}(\widetilde{u}, \widetilde{\sigma}), \bar{v}\right\rangle=0, \\
& \left\langle L_{2}(\widetilde{u}, \tilde{\sigma}), \bar{\tau}\right\rangle=0,
\end{aligned}
$$

which follows easily from changing the variables.

Finally, we'd like to prove that the recovered pressure is also "axisymmetric" in some sense. We look at $\partial_{\theta} p$ as a distribution in $D_{\varepsilon} \times(0,2 \pi)$. Let $q \in C_{0}^{1}\left(D_{\varepsilon} \times(0,2 \pi)\right)$ be arbitrary but fixed. Then if $v=T^{-1}(0,0, q)$, evidently $v \in C_{0}^{1}\left(\Omega_{\varepsilon}\right)$. The de-Rahm theory leads us to

$$
\left\langle L_{1}(\widetilde{u}, \tilde{\sigma}), v\right\rangle=\int_{\Omega_{\varepsilon}} p \operatorname{div} v
$$

where $(\tilde{u}, \tilde{\sigma})$ is the solution to problem $(3)$. Taking into account the definition of $v$ and the fact that $(\widetilde{u}, \tilde{\sigma}) \in V_{s}^{\prime} \times W_{s}^{\prime}$, we get that

$$
\left\langle L_{1}(\widetilde{u}, \tilde{\sigma}), v\right\rangle=0
$$


From (15) and (16) we derive

$$
0=\int_{\Omega_{\varepsilon}} p \operatorname{div} v=\int_{D_{\varepsilon} \times(0,2 \pi)} p \partial_{\theta} q,
$$

so that $\partial_{\theta} p=0$ in the distributional sense.

Let us summarize the results obtained in this section:

Proposition 4. Suppose that $f \in\left\{g \in \mathcal{X}_{s} \mid g_{\theta}=0\right\}$. Then the solution $(u, \sigma)$ to problem (1)-(2) belongs to the axisymmetric space $V_{s}^{\prime} \times W_{s}^{\prime}$. Moreover, the pressure satisfies $\partial_{\theta} p=0$ in the distributional sense.

\section{Numerical results}

This section is devoted to the numerical simulation of the fluid flows for the direct problem and the asymptotic problem. In particular in the axisymmetric case we analyse the behaviour of the solution of the 3D problem with respect to the solution of the asymptotic problem when the domain thickness becomes very small. When dealing with non-axisymmetric domains we present numerical results for the asymptotic model and discuss them qualitatively.

\subsection{Numerical methods}

The direct problem. Let us discuss the numerical simulation of the direct problem in the axisymmetric case. First, we rewrite the variational problem in cylindrical coordinates: Find $\left(u_{x}, u_{z}, \sigma_{x x}, \sigma_{x z}, \sigma_{z z}, \sigma_{\theta \theta}\right) \in V_{1}$ such that for all $\left(v_{x}, v_{z}, \tau_{x x}, \tau_{x z}, \tau_{z z}, \tau_{\theta \theta}\right) \in V_{1}$,

$$
\begin{aligned}
& \int_{D_{\varepsilon}}(1-r)\left(z\left(\partial_{x} u_{x} \partial_{x} v_{x}+\partial_{z} u_{x} \partial_{z} v_{x}+\partial_{x} u_{z} \partial_{x} v_{z}+\partial_{z} u_{z} \partial_{z} v_{z}\right)+\frac{u_{z} v_{z}}{z}\right) \\
&+ z\left(u_{x} \partial_{x}+u_{z} \partial_{z}\right)\left(u_{x} v_{x}+u_{z} v_{z}\right) \\
&+ z\left(\sigma_{x x} \partial_{x} v_{x}+\sigma_{x z}\left(\partial_{x} v_{z}+\partial_{z} v_{x}\right)+\sigma_{z z} \partial_{z} v_{z}\right)+\sigma_{\theta \theta} v_{z}=\int_{D_{\varepsilon}} z f v_{x} \\
& \int_{D_{\varepsilon}} z\left(u_{x} \partial_{x}+u_{z} \partial_{z}\right)\left(\sigma_{x x} \tau_{x x}+2 \sigma_{x z} \tau_{x z}+\sigma_{z z} \tau_{z z}+\sigma_{\theta \theta} \tau_{\theta \theta}\right) \\
&+z\left(\partial_{x} u_{z}-\partial_{z} u_{x}\right)\left(\sigma_{x z}\left(\tau_{z z}-\tau_{x x}\right)-\tau_{x z}\left(\sigma_{z z}-\sigma_{x x}\right)\right) \\
&+ z\left(\sigma_{x x} \tau_{x x}+2 \sigma_{x z} \tau_{x z}+\sigma_{z z} \tau_{z z}+\sigma_{\theta \theta} \tau_{\theta \theta}\right) \\
&+ \varepsilon^{2}\left(z \left(\partial_{x} \sigma_{x x} \partial_{x} \tau_{x x}+\partial_{z} \sigma_{x x} \partial_{z} \tau_{x x}+2 \partial_{x} \sigma_{x z} \partial_{x} \tau_{x z}+2 \partial_{z} \sigma_{x z} \partial_{z} \tau_{x z}\right.\right. \\
&\left.+\partial_{x} \sigma_{z z} \partial_{x} \tau_{z z}+\partial_{z} \sigma_{z z} \partial_{z} \tau_{z z}+\partial_{x} \sigma_{\theta \theta} \partial_{x} \tau_{\theta \theta}+\partial_{z} \sigma_{\theta \theta} \partial_{z} \tau_{\theta \theta}\right) \\
&\left.+\frac{\sigma_{x z} \tau_{x z}+\sigma_{z z} \tau_{z z}+\sigma_{\theta \theta} \tau_{\theta \theta}}{z}\right) \\
&= \int_{D_{\varepsilon}} z\left(\tau_{x x} \partial_{x} u_{x}+\tau_{x z}\left(\partial_{x} u_{z}+\partial_{z} u_{x}\right)+\tau_{z z} \partial_{z} u_{z}\right)+\tau_{\theta \theta} u_{z}
\end{aligned}
$$

where $V_{1}$ is an appropriate variational space.

The boundary conditions translate to

$$
\begin{cases}u=0, \quad \sigma=0 & \text { on } \gamma_{\varepsilon} \\ u_{z}=\sigma_{x z}=\sigma_{z z}=\sigma_{\theta \theta}=0 & \text { on } C \\ u, \sigma \text { and } p \text { are } x_{1} \text { periodic. } & \end{cases}
$$

where we have written the lower and the upper part of the boundary of $D_{\varepsilon}$ as

$$
C=\{(x, 0) \mid x \in[0,1]\}, \quad \gamma_{\varepsilon}=\{(x, \varepsilon h(x)) \mid x \in[0,1]\} .
$$


Following equations (AS1), (AS2) we define the following operators:

$$
\begin{aligned}
A_{1}(u, v)= & \int_{D_{\varepsilon}}(1-r)\left(z\left(\partial_{x} u_{x} \partial_{x} v_{x}+\partial_{z} u_{x} \partial_{z} v_{x}+\partial_{x} u_{z} \partial_{x} v_{z}+\partial_{z} u_{z} \partial_{z} v_{z}\right)+\frac{u_{z} v_{z}}{z}\right) \\
A_{2}(\sigma, v)= & \int_{D_{\varepsilon}} z\left(\sigma_{x x} \partial_{x} v_{x}+\sigma_{x z}\left(\partial_{x} v_{z}+\partial_{z} v_{x}\right)+\sigma_{z z} \partial_{z} v_{z}\right)+\sigma_{\theta \theta} v_{z} \\
A_{3}(\sigma, \tau)= & \int_{D_{\varepsilon}} z\left(\sigma_{x x} \tau_{x x}+2 \sigma_{x z} \tau_{x z}+\sigma_{z z} \tau_{z z}+\sigma_{\theta \theta} \tau_{\theta \theta}\right) \\
& \quad+\varepsilon^{2}\left(z \left(\partial_{x} \sigma_{x x} \partial_{x} \tau_{x x}+\partial_{z} \sigma_{x x} \partial_{z} \tau_{x x}+2 \partial_{x} \sigma_{x z} \partial_{x} \tau_{x z}\right.\right. \\
& \left.\quad+2 \partial_{z} \sigma_{x z} \partial_{z} \tau_{x z}+\partial_{x} \sigma_{z z} \partial_{x} \tau_{z z}+\partial_{z} \sigma_{z z} \partial_{z} \tau_{z z}+\partial_{x} \sigma_{\theta \theta} \partial_{x} \tau_{\theta \theta}+\partial_{z} \sigma_{\theta \theta} \partial_{z} \tau_{\theta \theta}\right) \\
& \left.\quad+\frac{\sigma_{x z} \tau_{x z}+\sigma_{z z} \tau_{z z}+\sigma_{\theta \theta} \tau_{\theta \theta}}{z}\right) \\
A_{4}(u, \tau)= & \int_{D_{\varepsilon}} z\left(\tau_{x x} \partial_{x} u_{x}+\tau_{x z}\left(\partial_{x} u_{z}+\partial_{z} u_{x}\right)+\tau_{z z} \partial_{z} u_{z}\right)+\tau_{\theta \theta} u_{z} \\
N_{1}(u, w, v)= & \int_{D_{\varepsilon}} z\left(u_{x} \partial_{x}+u_{z} \partial_{z}\right)\left(w_{x} v_{x}+w_{z} v_{z}\right) \\
N_{2}(u, \sigma, \tau)= & \int_{D_{\varepsilon}} z\left(u_{x} \partial_{x}+u_{z} \partial_{z}\right)\left(\sigma_{x x} \tau_{x x}+2 \sigma_{x z} \tau_{x z}+\sigma_{z z} \tau_{z z}+\sigma_{\theta \theta} \tau_{\theta \theta}\right) \\
N_{3}(u, \sigma, \tau)= & \int_{D_{\varepsilon}} z\left(\partial_{x} u_{z}-\partial_{z} u_{x}\right)\left(\sigma_{x z}\left(\tau_{z z}-\tau_{x x}\right)-\tau_{x z}\left(\sigma_{z z}-\sigma_{x x}\right)\right) \\
l(v)= & \int_{D_{\varepsilon}} z f v_{x}+p_{d} \int_{C_{0}^{\varepsilon}} v_{x},
\end{aligned}
$$

which leads us to consider the following discrete problem:

$$
\left\{\begin{aligned}
& \text { Find }\left(u_{h}, \sigma_{h}, p_{h}\right) \in V_{h} \times W_{h} \times Q_{h} \text { such that, for all }\left(v_{h}, \tau_{h}, q_{h}\right) \in V_{h} \times W_{h} \times Q_{h}, \\
& A_{1}\left(u_{h}, v_{h}\right)+N_{1}\left(u_{h}, u_{h}, v_{h}\right)+A_{2}\left(\sigma_{h}, v_{h}\right)+B\left(p_{h}, v_{h}\right)=l\left(v_{h}\right) \\
& B\left(q_{h}, u_{h}\right)=0, \\
& N_{2}\left(u_{h}, \sigma_{h}, \tau_{h}\right)+N_{3}\left(u_{h}, \sigma_{h}, \tau_{h}\right)+A_{3}\left(\sigma_{h}, \tau_{h}\right)=A_{4}\left(u_{h}, \tau_{h}\right),
\end{aligned}\right.
$$

where $V_{h}, W_{h}, Q_{h}$ are appropriate finite-dimensional subspaces and $B(p, v)=\int_{D_{\varepsilon}} p$ div $v$. The numerical analysis of the above problem is outside the scope of this paper - we refer to [3] for a detailed study of the axisymmetric Navier-Stokes system. Here, we shall present two iterative algorithms for solving the problem (17) by linearization. We drop the subscript $h$ for convenience. Note that the way to address the computation of a nonlinear system usually relies on an iterative process based upon a linearized problem. In the case of the Oldroyd system, we present two different methods:

Method I. Start with $u^{0}=0, \sigma^{0}=0$. Define $u^{n+1}, \sigma^{n+1}$ and $p^{n+1}$ by

$$
\left\{\begin{aligned}
& \text { Find }\left(u^{n+1}, \sigma^{n+1}, p^{n+1}\right) \in V_{h} \times W_{h} \times Q_{h} \text { such that, for all }(v, \tau, q) \in V_{h} \times W_{h} \times Q_{h}, \\
& A_{1}\left(u^{n+1}, v\right)+N_{1}\left(u^{n}, u^{n+1}, v\right)+A_{2}\left(\sigma^{n}, v\right)+B\left(p^{n+1}, v\right)=l(v) \\
& B\left(q, u^{n+1}\right)=0 \\
& N_{2}\left(u^{n}, \sigma^{n+1}, \tau\right)+N_{3}\left(u^{n+1}, \sigma^{n+1}, \tau\right)+A_{3}\left(\sigma^{n+1}, \tau\right)=A_{4}\left(u^{n+1}, \tau\right) .
\end{aligned}\right.
$$

Method II. Start with $u^{0}=0, \sigma^{0}=0$. Define $u^{n+1}, \sigma^{n+1}$ and $p^{n+1}$ by

$$
\left\{\begin{aligned}
\text { Find }\left(u^{n+1}, \sigma^{n+1}, p^{n+1}\right) \in V_{h} \times W_{h} \times Q_{h} \text { such that, for all } & (v, \tau, q) \in V_{h} \times W_{h} \times Q_{h}, \\
A_{1}\left(u^{n+1}, v\right)+N_{1}\left(u^{n}, u^{n+1}, v\right)+A_{2}\left(\sigma^{n+1}, v\right)+B\left(p^{n+1}, v\right) & =l(v) \\
B\left(q, u^{n+1}\right) & =0 \\
N_{2}\left(u^{n}, \sigma^{n+1}, \tau\right)+N_{3}\left(u^{n}, \sigma^{n+1}, \tau\right)+A_{3}\left(\sigma^{n+1}, \tau\right) & =A_{4}\left(u^{n+1}, \tau\right) .
\end{aligned}\right.
$$

In both cases, the solution of the problem is defined as $\left(u^{\infty}, \sigma^{\infty}, p^{\infty}\right)$. In practice the algorithm is stopped by a criterion based upon the error related to the convergence process. Let us comment the differences between the two algorithms: 
- Note the subtle but crucial difference. While the first method involves splitting the system and treating them separately, the second one solves the system as a whole. In detail, Method I is a splitting process which consists in defining $\left(u^{n+1}, p^{n+1}\right)$ as the solution of a generalized Stokes problem and then update $\sigma^{n+1}$ with the linearized constitutive equation, whereas Method II defines $\left(u^{n+1}, \sigma^{n+1}, p^{n+1}\right)$ as the solution of a linear mixed problem. In both methods, quadratic nonlinear terms are treated in a classical way which consists in preserving the main unknown in the equation (the velocity field in the momentum equation, the extra-stress in the constitutive equation).

- The first method is computationally faster but has the disadvantage of not converging for $r \geq 8 / 9$. As a consequence Method I is generally privileged when the fluid is not too elastic; but computations with large values of $r$ are possible.

- In both cases, the computation of the numerical solution may rely on standard finite element solvers which only require the determination of the solution of well-posed elliptic linear problems. In simulations, we use $\mathbb{P}^{2}$ elements for the velocity field, $\mathbb{P}^{1}$ for the pressure (ensuring the inf-sup condition at the discrete level) and $\mathbb{P}^{1}$ elements for the extra-stress.

The asymptotic model. We aim at computing the main order of the asymptotic expansion:

$$
\widetilde{u} \sim \varepsilon^{2}\left(\widetilde{u}_{1}^{0}, 0,0\right), \quad \widetilde{p} \sim \widetilde{p}^{0}, \quad \widetilde{\sigma} \sim \varepsilon\left(\begin{array}{ccc}
0 & \widetilde{\sigma}_{12}^{0} & \widetilde{\sigma}_{13}^{0} \\
\widetilde{\sigma}_{12}^{0} & 0 & 0 \\
\widetilde{\sigma}_{13}^{0} & 0 & 0
\end{array}\right)
$$

We shall use a superposition principle to compute the solution of the asymptotic problem:

i. For all $x_{1} \in(0,1)$, define $\widetilde{v}_{1}\left(x_{1}, \cdot\right)$ as the unique solution of the bidimensional scalar problem

$$
\mathrm{P}_{1}\left(x_{1}\right)\left\{\begin{array}{c}
\text { Find } \widetilde{v}\left(x_{1}, \cdot\right) \in H_{0}^{1}\left(S\left(x_{1}\right)\right) \text { such that, for all } v \in H_{0}^{1}\left(S\left(x_{1}\right)\right), \\
(1-r) \int_{S\left(x_{1}\right)} \nabla_{k} \widetilde{v}\left(x_{1}, \cdot\right) \cdot \nabla_{k} v=\int_{S\left(x_{1}\right)} \widetilde{f}_{1}\left(x_{1}, \cdot\right) v
\end{array}\right.
$$

where $\nabla_{k} v=\left(\partial_{2} v, \partial_{3} v\right)^{t}$. In practice, we solve these $2 \mathrm{D}$ problems by using $\mathbb{P}^{1}$ elements and compute

$$
\gamma_{1}\left(x_{1}\right):=\int_{S\left(x_{1}\right)} \partial_{1} \widetilde{v}_{1}\left(x_{1}, \cdot\right)
$$

by means of a simple integration.

ii. For all $x_{1} \in(0,1)$, define $\left(\widetilde{v}_{2},\left(\widetilde{\tau}_{2}, \widetilde{\tau}_{3}\right)\right)\left(x_{1}, \cdot\right)$ as the unique solution of the bidimensional problem

$$
\mathrm{P}_{2}\left(x_{1}\right)\left\{\begin{aligned}
\text { Find }(\widetilde{v}, \widetilde{\tau})\left(x_{1}, \cdot\right) & \in\left[H_{0}^{1}\left(S\left(x_{1}\right)\right)\right]^{3} \text { such that, for all }(v, \tau) \in\left[H_{0}^{1}\left(S\left(x_{1}\right)\right)\right]^{3}, \\
(1-r) \int_{S\left(x_{1}\right)} \nabla_{k} \widetilde{v}\left(x_{1}, \cdot\right) \cdot \nabla_{k} v+\int_{S\left(x_{1}\right)} \widetilde{\tau} \cdot \nabla_{k} v & =-\int_{S\left(x_{1}\right)} v, \\
\int_{S\left(x_{1}\right)} \nabla_{k} \widetilde{\tau}\left(x_{1}, \cdot\right) \cdot \nabla_{k} \tau+\int_{S\left(x_{1}\right)} \widetilde{\tau}\left(x_{1}, \cdot\right) \tau-r \int_{S\left(x_{1}\right)} \nabla_{k} \widetilde{v}\left(x_{1}, \cdot\right) \cdot \tau & =0
\end{aligned}\right.
$$

and define

$$
\gamma_{2}\left(x_{1}\right):=\int_{S\left(x_{1}\right)} \partial_{1} \widetilde{v}_{2}\left(x_{1}, \cdot\right)
$$

These $2 \mathrm{D}$ problems are solved using $\mathbb{P}^{1}$ elements for both the velocity and the elastic stress.

iii. Define $\widetilde{p}^{0}$ as the unique solution of

$$
-\partial_{1}\left(\gamma_{2}\left(x_{1}\right) \partial_{1} \widetilde{p}^{0}\left(x_{1}\right)\right)=\gamma_{1}\left(x_{1}\right) \quad \text { and } \quad \widetilde{p}^{0}(0)-\widetilde{p}^{0}(1)=p_{d}
$$

by means of simple integrations.

iv. Define $\left(\widetilde{u}_{1}^{0}, \widetilde{\sigma}_{12}^{0}, \widetilde{\sigma}_{13}^{0}\right)$ as

$$
\left(\widetilde{u}_{1}^{0}, \widetilde{\sigma}_{12}^{0}, \widetilde{\sigma}_{13}^{0}\right)=\left(\widetilde{v}_{1}+\partial_{1} \widetilde{p}^{0} \widetilde{v}_{2}, \partial_{1} \widetilde{p}^{0} \widetilde{\tau}_{2}, \partial_{1} \widetilde{p}^{0} \widetilde{\tau}_{3}\right)
$$


Let us give some details on the computation of the numerical solution: Steps i. and ii. can be done with any standard 2D finite element solver (with $\mathbb{P}^{1}$ finite elements for instance) and only require the repetition of the computation for many positions of $x_{1}$. However note that this repetition can be drastically reduced if $x_{1} \mapsto S\left(x_{1}\right)$ and $x_{1} \mapsto \widetilde{f}_{1}\left(x_{1}, \cdot\right)$ do not vary on a significant range of the coordinate $x_{1} \in(0,1)$. Besides computations in Steps i. and ii. can be easily driven in parallel. Finally item iii. can be done by performing simple numerical integrations.

\subsection{Numerical validation in the axisymmetric case}

Let us now consider specific examples on which we will base our numerical simulations. Two axisymmetric geometries are investigated:

- Geometry (I) is a thin axisymmetric tube with a constriction step whose section $D_{\varepsilon}$ is completely described by the boundary (see the picture below).

$$
\begin{aligned}
& C_{0}^{\varepsilon}=\{(0, z) \mid z \in(0, \varepsilon)\}, \quad C_{1}^{\varepsilon}=\{(1, z) \mid z \in(0, \varepsilon)\}, \\
& C=\{(x, 0) \mid x \in(0,1)\} \quad \text { and } \quad \gamma_{\varepsilon}=\{(x, \varepsilon h(x)) \mid x \in(0,1)\},
\end{aligned}
$$

where

$$
h(x)=\left\{\begin{aligned}
1, & x \in(0.0,0.4), \\
1.5-1.25 \cdot x, & x \in(0.4,0.6), \\
1, & x \in(0.6,1.0) .
\end{aligned}\right.
$$

Dirichlet conditions are prescribed on $\gamma_{\varepsilon}$ and periodic conditions are prescribed on $C_{0}^{\varepsilon}$ and $C_{1}^{\varepsilon}$ for both the velocity and the elastic stress.

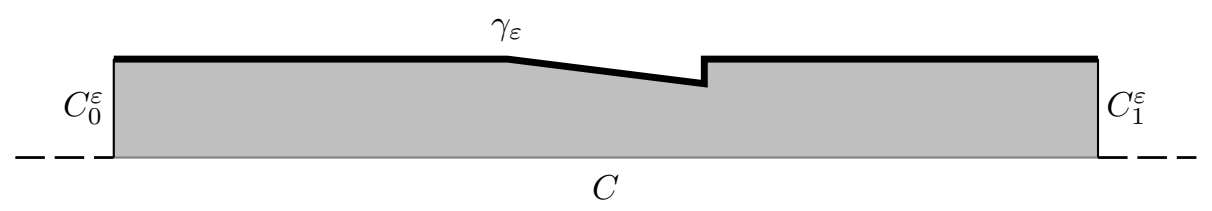

- Geometry (II) is a thin axisymmetric tube with an inner obstacle whose section $D_{\varepsilon}$ is completely described by the boundary (see the picture below). Dirichlet conditions are prescribed on $\gamma_{\varepsilon}$ and free outlet conditions are prescribed on $C_{0}^{\varepsilon}$ and $C_{1}^{\varepsilon}$ for both the velocity and the elastic stress.

$$
\begin{aligned}
& C_{0}^{\varepsilon}=\{(0, z) \mid z \in(0, \varepsilon)\}, \quad C_{1}^{\varepsilon}=\{(1, z) \mid z \in(0.75 \varepsilon, \varepsilon)\}, \\
& C=\{(x, 0) \mid x \in(0,0.25)\} \quad \text { and } \quad \gamma_{\varepsilon}=\{(x, \varepsilon) \mid x \in(0,1)\} \cup\{(0.25, y) \mid y \in(0, \varepsilon \beta)\} \cup\{(x, \varepsilon) \mid x \in(0,1)\},
\end{aligned}
$$

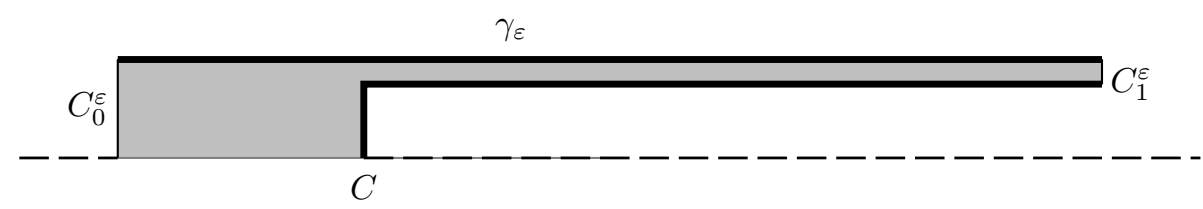

Note that these geometries lack the required regularity of our analysis. Nevertheless numerical simulations allow us to overcome this issue. We use everywhere the model parameters (elasticity parameter, source term, pressure drop):

$$
r=0.5, \quad \tilde{f}=0, \quad p_{d}=1 .
$$

Computations have been led:

- with the direct model for $\varepsilon \in\{0.3, \ldots, 0.1\}$ in which case the solution is denoted $\left(u_{\varepsilon}, \sigma_{\varepsilon}, p_{\varepsilon}\right)$. Furthermore in the geometry (I) we define $\bar{p}_{\varepsilon}$ as the average pressure along the $x_{1}$-axis, i.e.

$$
\bar{p}_{\varepsilon}\left(x_{1}\right)=\frac{1}{\varepsilon h\left(x_{1}\right)} \int_{0}^{\varepsilon h\left(x_{1}\right)} p\left(x_{1}, \cdot\right) .
$$

A similar definition of the average pressure along the $x$-axis is done for the geometry (II). 
- with the asymptotic model, in which case the solution is denoted $(\tilde{u}, \tilde{\sigma}, \tilde{p})$.

Remark 3. Let us give some details on how to solve the asymptotic solution in the case of geometries (I) or (II):

- The computation of the solution of the asymptotic model only requires to solve elliptic problems

- on $2 D$ disks for geometry (I), see Figure 8 and 9 ;

- on $2 D$ disks for $x_{1} \in(0,0.25)$, see Figure 10, and on $2 D$ annuli for $x_{1} \in(0.25,1)$, see Figure 11 .

- Step $i$. is straightforward if the source term satisfies $\tilde{f}=0$ (as assumed in our computations): in this case the solution $\tilde{v}_{1}\left(x_{1}, \cdot\right)$ of problem $\mathrm{P}_{1}\left(x_{1}\right)$ reduces to 0 for any $x_{1}$ and, as a consequence, $\gamma_{1}\left(x_{1}\right)=0$ for any $x_{1}$.

- In step ii., solving problems $\mathrm{P}_{2}\left(x_{1}\right)$ for $x_{1} \in(0,1)$ can be done as follows:

- for geometry $(I)$, solving problems $\mathrm{P}_{2}\left(x_{1}\right)$ for $x_{1} \in(0,0.4) \cup(0.6,1)$ reduces to one single resolution as $x_{1} \mapsto S\left(x_{1}\right)$ does not vary on this range of coordinates; solving problems $\mathrm{P}_{2}\left(x_{1}\right)$ for $x_{1} \in(0.4,0.6)$ cannot be reduced similarly and, in practice, the number of resolution for this range of axial coordinates depends on the discretization number of the axial coordinate.

- for geometry (II), the resolution of two elliptic problems is only required for the whole range of axial coordinates $x_{1}$ : indeed problem $\mathrm{P}_{2}\left(x_{1}\right)$ is solved once on a disk for any $x_{1} \in(0,0.25)$ and problem $\mathrm{P}_{2}\left(x_{1}\right)$ is solved once on $a$ annulus for any $x_{1} \in(0.25,1)$.

- As $\gamma_{1} \equiv 0$, the pressure field is defined as (as $\left.p_{d}=1\right)$ :

$$
\tilde{p}\left(x_{1}\right)=-\frac{\int_{0}^{x_{1}} \gamma_{2}}{\int_{0}^{1} \gamma_{2}}
$$

which can be computed by means of numerical integrations.

Figures 1-4 provide the numerical results obtained with the direct model for the velocity field, the pressure field, the pressure field and the components of the elastic extra-stress. In order to compare the direct model to the asymptotic one, we focus on the pressure profile: we compare the average pressure for various values of $\varepsilon$ to the asymptotic pressure $\tilde{p}$, see Figures 5 and 6 , and we numerically obtain:

$$
\left\|\bar{p}_{\varepsilon}-\tilde{p}\right\|_{L^{2}(0,1)}=\mathcal{O}(\varepsilon),
$$

see in particular Figure 7. 

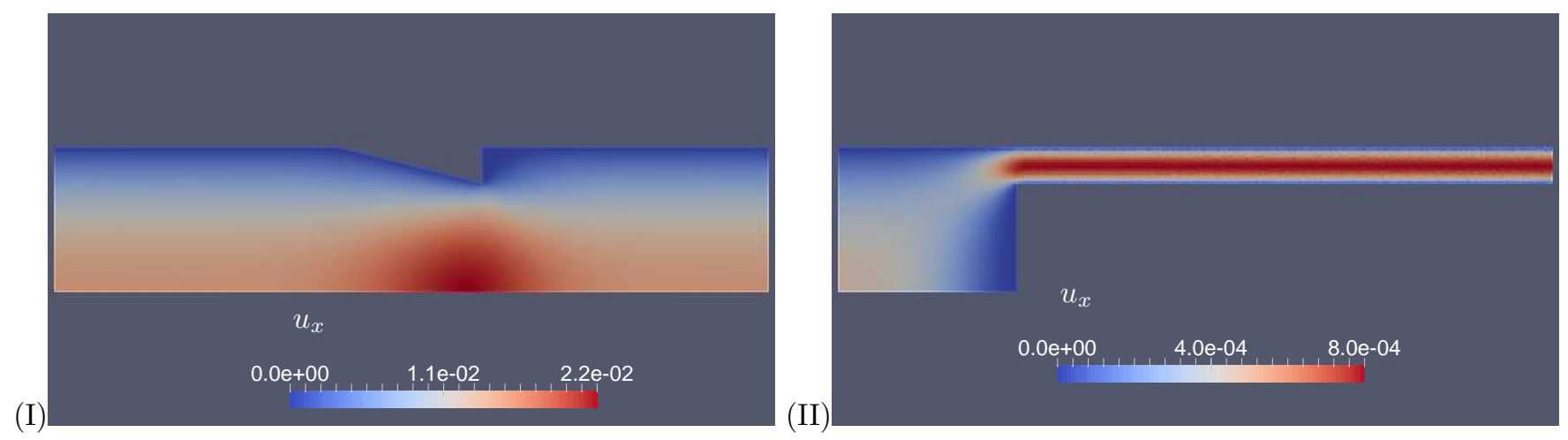

Figure 1: Geometry (I) or (II) with $\varepsilon=0.2$ : axial component of the velocity field.
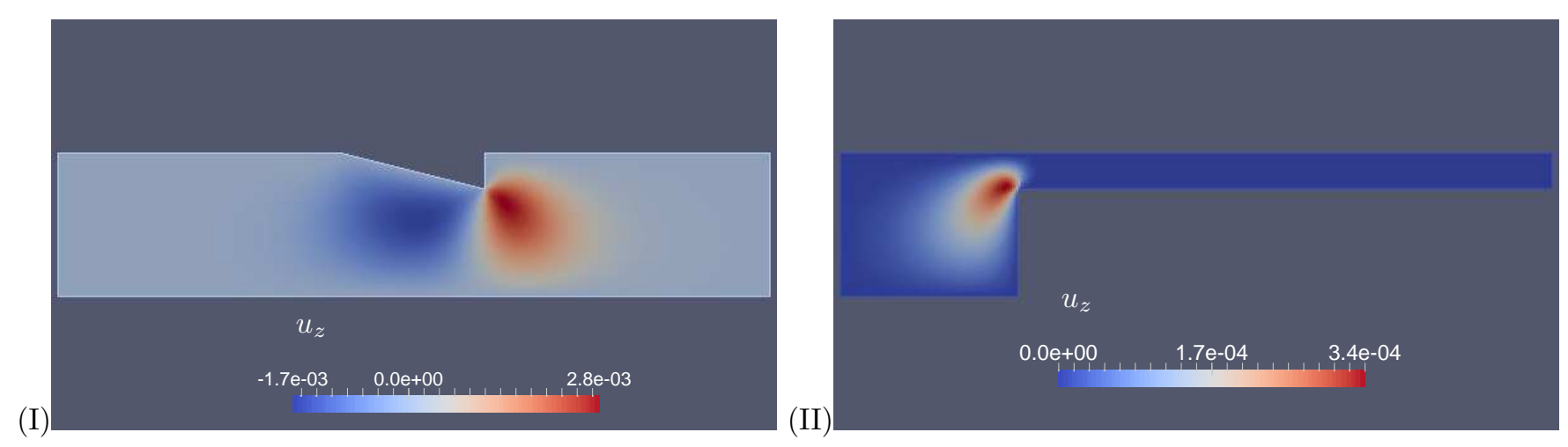

Figure 2: Geometry (I) or (II) with $\varepsilon=0.2$ : radial component of the velocity field.
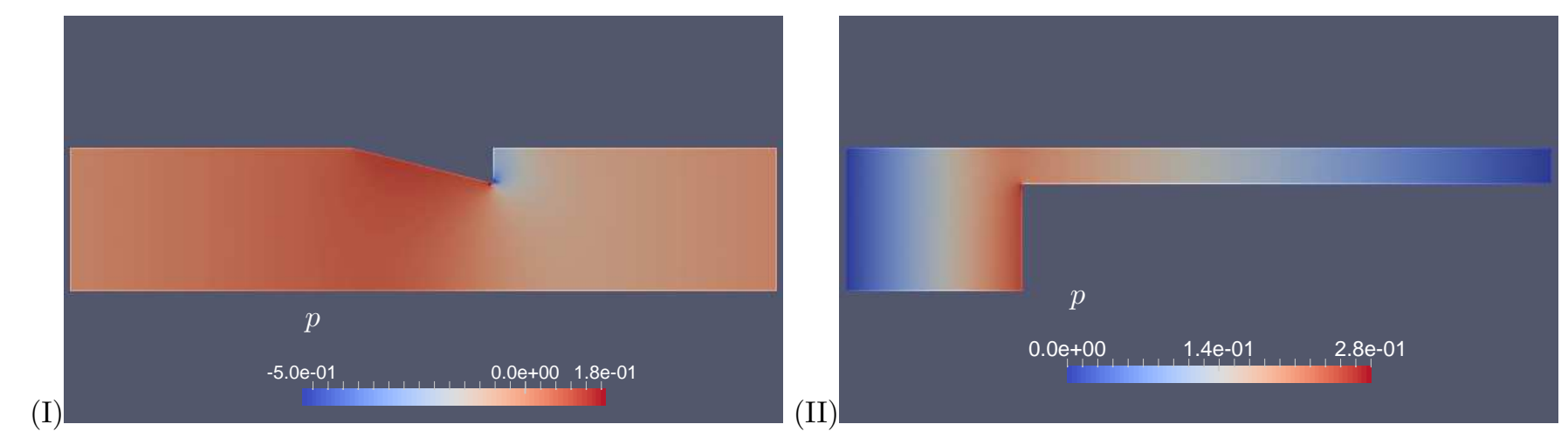

Figure 3: Geometry (I) or (II) with $\varepsilon=0.2$ : pressure field. Note that the linear profile $(x, y, z) \mapsto-x$ due to the pressure drop $p_{d}=1$ has been removed in the above results. 


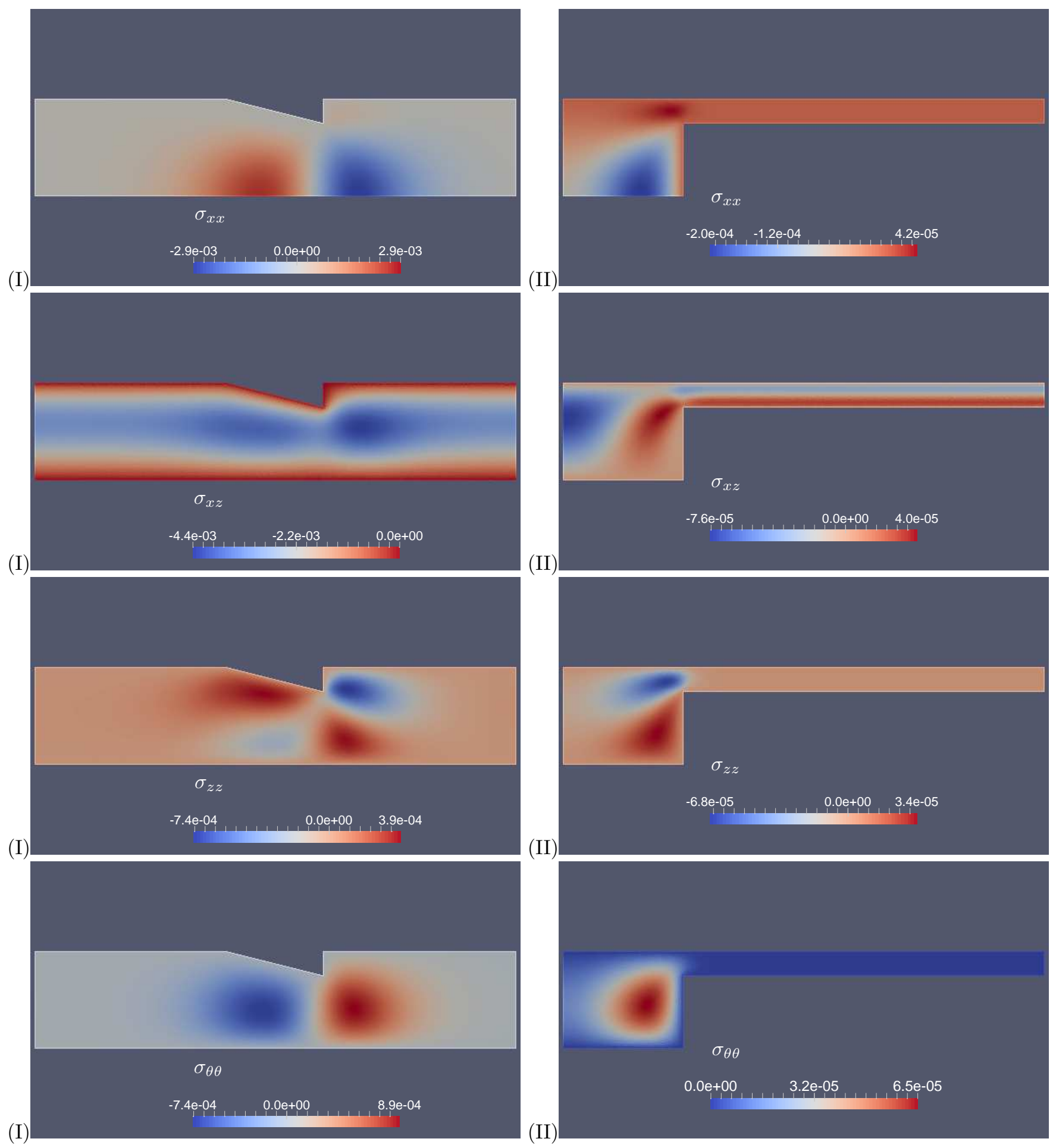

Figure 4: Geometry (I) or (II) with $\varepsilon=0.2$ : components of the elastic extra-stress. 


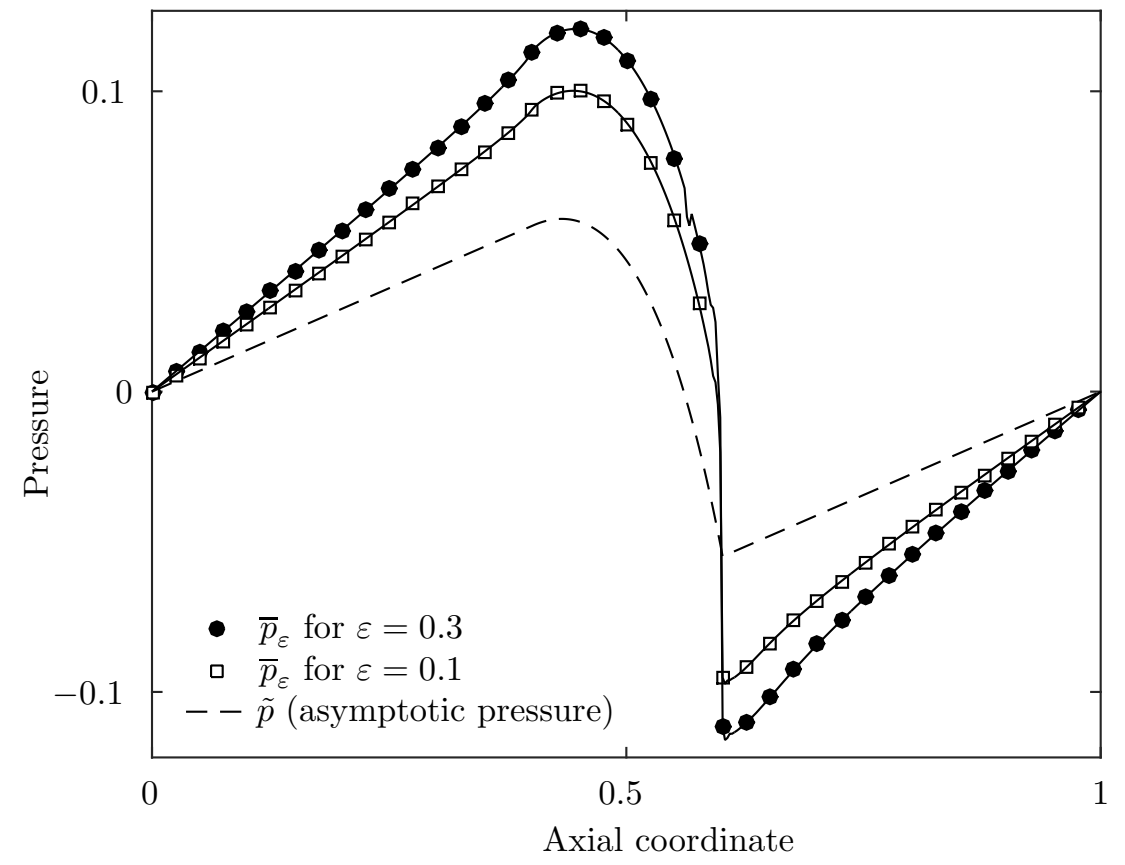

Figure 5: Geometry (I): pressure profiles for various values of the radius magnitude and in the asymptotic case (note that the linear contribution $x \mapsto-x$ has been removed).

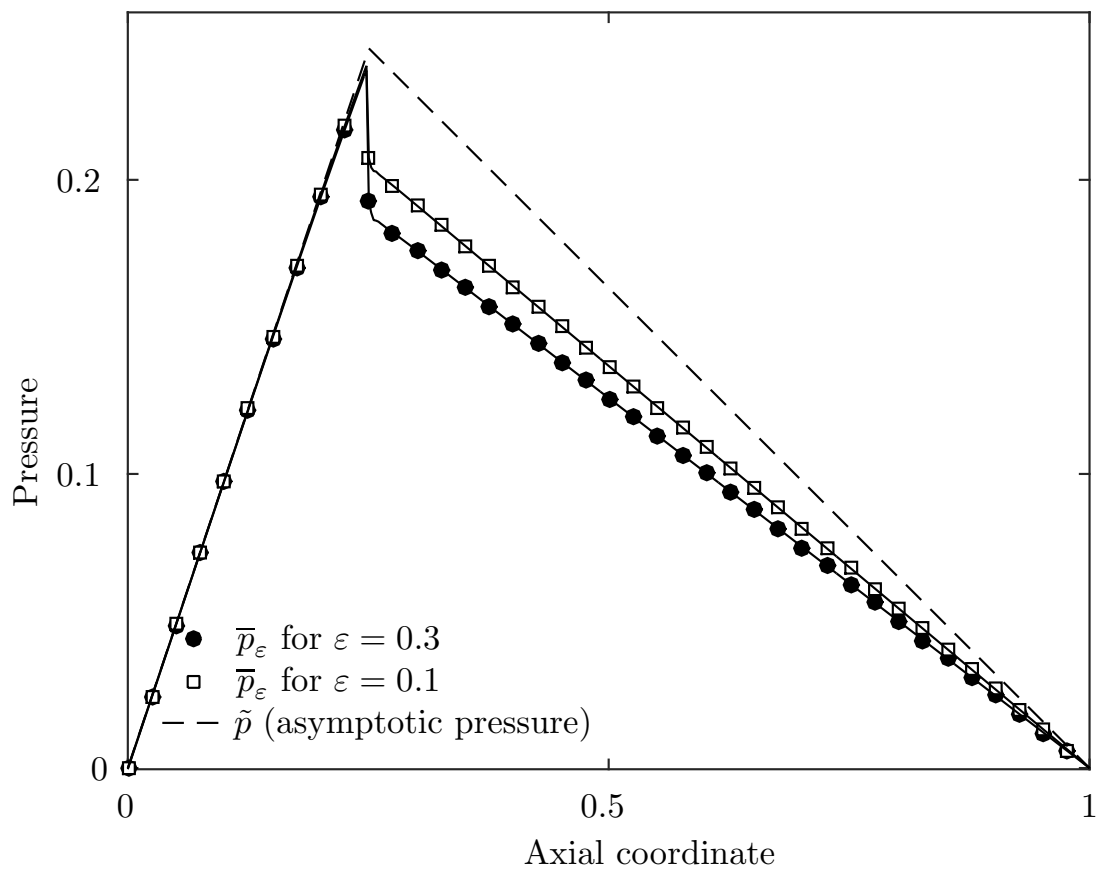

Figure 6: Geometry (II): pressure profiles for various values of the radius magnitude and in the asymptotic case (note that the linear contribution $x \mapsto-x$ has been removed). 


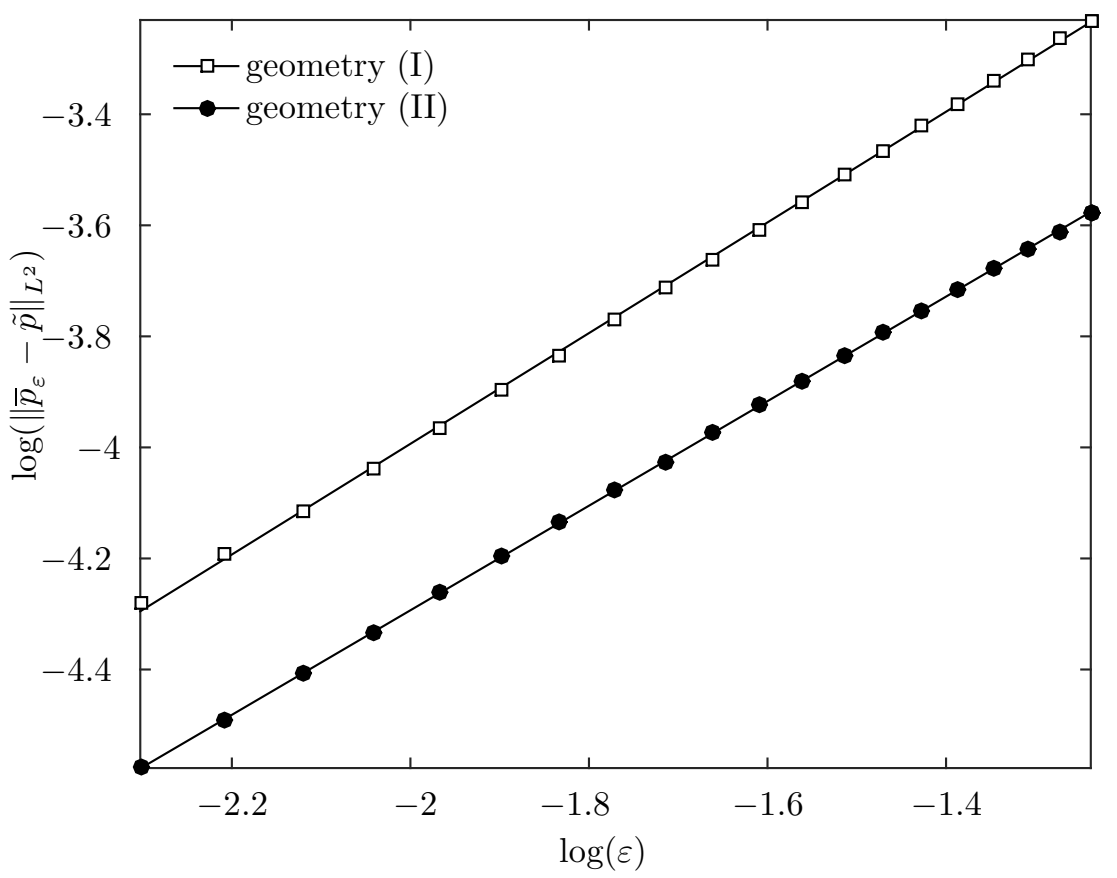

Figure 7: Geometry (II): convergence of the average pressure $\bar{p}_{\varepsilon}$ to the asymptotic pressure $\tilde{p}$ with respect to the small parameter $\varepsilon$.

\subsection{The asymptotic model in the non-axisymmetric case}

We consider the case of a non-axisymmetric domain, namely a cylinder with an upper constriction referred as geometry (III), see Figure 12:

- for $x_{1} \in(0,0.4) \cup(0.6,1)$, the section $S\left(x_{1}\right)$ is the disk of radius 1 ;

- for $x_{1} \in(0.4,0.6)$, the section $S\left(x_{1}\right)$ is modified by removing from the unit disk the upper part $[z>1-K]$ with $K=0.15$.

We also consider the case of an axisymmetric domain, namely a cylinder with a constriction referred as geometry (IV), see Figure 13:

- for $x_{1} \in(0,0.4) \cup(0.6,1)$, the section $S\left(x_{1}\right)$ is the disk of radius 1 ;

- for $x_{1} \in(0.4,0.6)$, the section $S\left(x_{1}\right)$ is the disk of radius $1-K$ with $K=0.15$.

Geometries (III) and (IV) only differ in the constriction part, namely for $x_{1} \in(0.4,0.6)$ : in geometry (III) the constriction of magnitude $K$ in the upper part whereas, in geometry (IV), the constriction of magnitude $K$ is axisymmetric.

Numerical simulations provide the asymptotic pressure, velocity field and elastic extra-stress tensor:

$$
\widetilde{u} \sim \varepsilon^{2}\left(\widetilde{u}_{1}^{0}, 0,0\right), \quad \widetilde{p} \sim \widetilde{p}^{0}, \quad \widetilde{\sigma} \sim \varepsilon\left(\begin{array}{ccc}
0 & \widetilde{\sigma}_{12}^{0} & \widetilde{\sigma}_{13}^{0} \\
\widetilde{\sigma}_{12}^{0} & 0 & 0 \\
\widetilde{\sigma}_{13}^{0} & 0 & 0
\end{array}\right) .
$$

Let us comment the results:

- Figure 14 focuses on the pressure profile for geometries (III) and (IV). Pressure variations are more important in geometry (IV) whose constriction magnitude is larger than in geometry (III).

- Figures 15 and 16 present the velocity and the components of the elastic stress tensor at $x_{1}=0.25$ (in the regular area) and $x_{1}=0.50$ (in the constricted area) for geometry (III). 

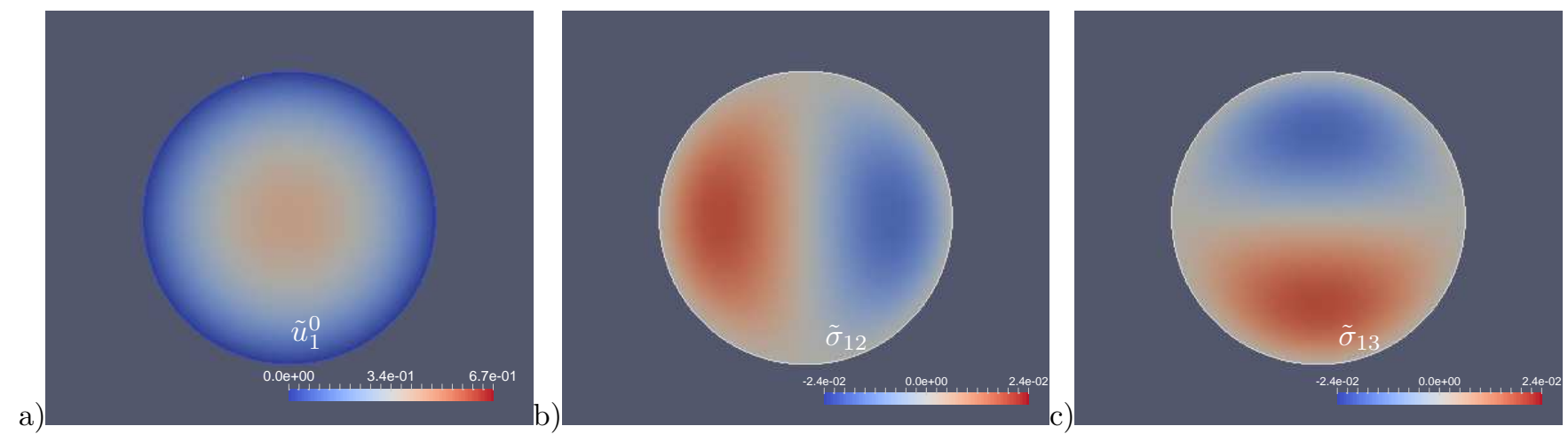

Figure 8: Geometry (I): normalized fields at $x_{1}=0.25$ : a) velocity profile $\tilde{u}_{1}^{0}$, b) extra-stress $\tilde{\sigma}_{12}$, c) extra-stress $\tilde{\sigma}_{13}$.
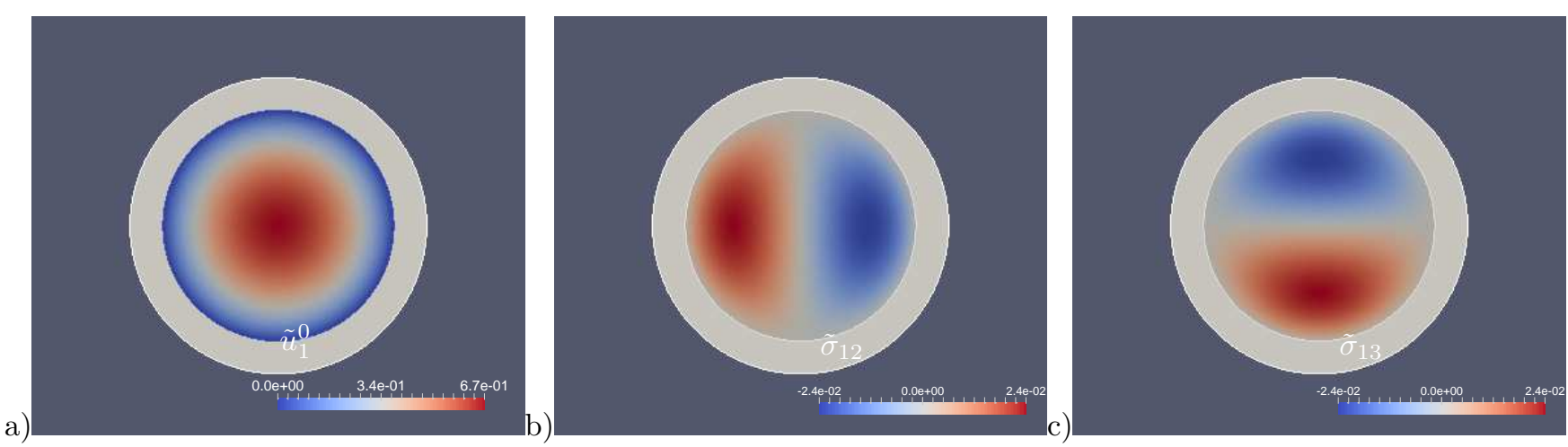

Figure 9: Geometry (I): normalized fields at $x_{1}=0.58$ : a) velocity profile $\tilde{u}_{1}^{0}$, b) extra-stress $\tilde{\sigma}_{12}$, c) extra-stress $\tilde{\sigma}_{13}$. 

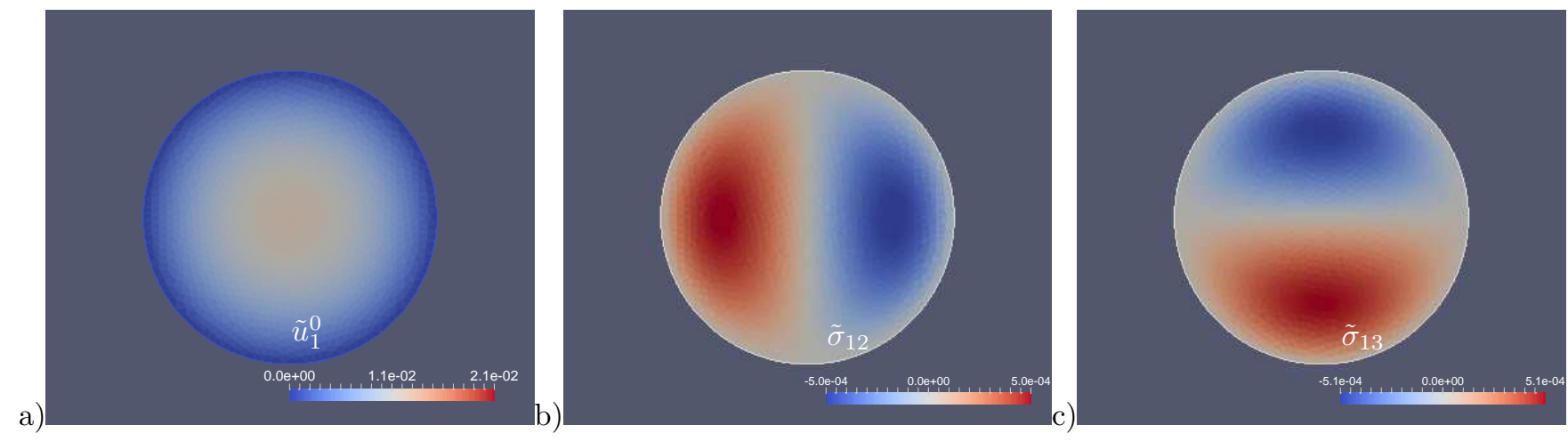

Figure 10: Geometry (II): normalized fields at $x_{1}=0.125$ : a) velocity profile $\tilde{u}_{1}^{0}$, b) extra-stress $\tilde{\sigma}_{12}$, c) extra-stress $\tilde{\sigma}_{13}$.
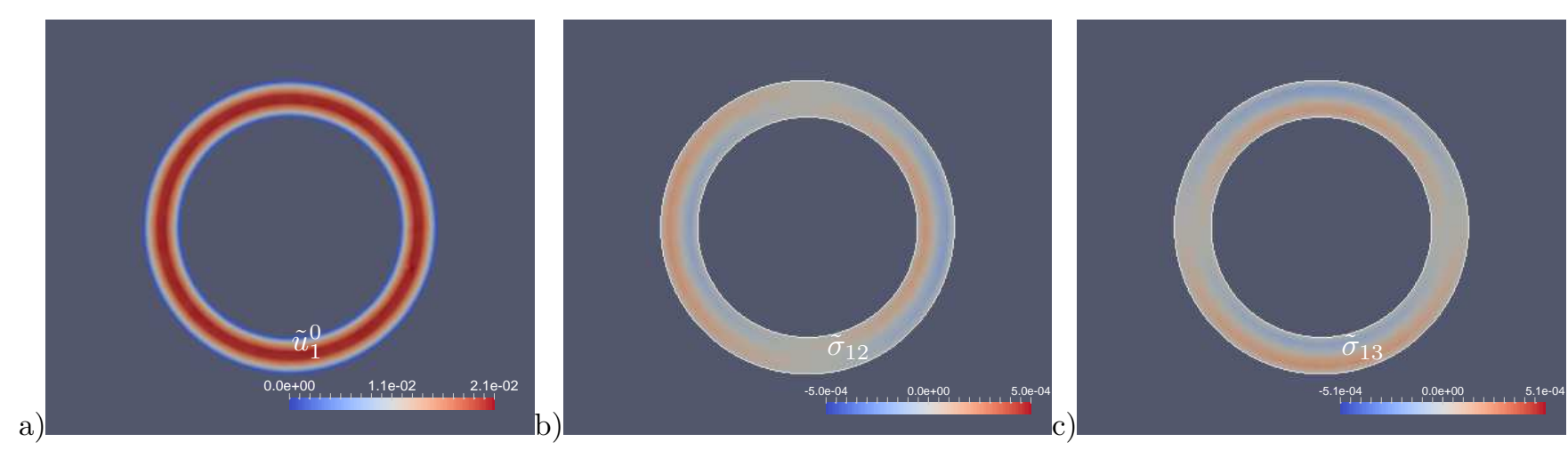

Figure 11: Geometry (II): normalized fields at $x_{1}=0.500$ : a) velocity profile $\tilde{u}_{1}^{0}$, b) extra-stress $\tilde{\sigma}_{12}$, c) extra-stress $\tilde{\sigma}_{13}$. 


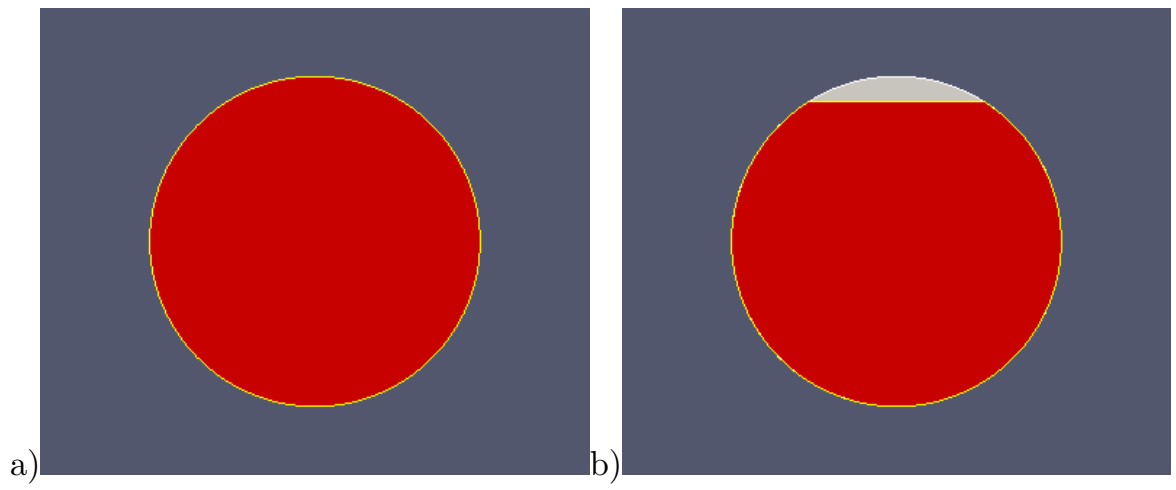

Figure 12: Geometry (III): a) section $S\left(x_{1}\right)$ for $x_{1} \in(0,0.4) \cup(0.6,1)$, b) section $S\left(x_{1}\right)$ for $x_{1} \in(0.4,0.6)$.

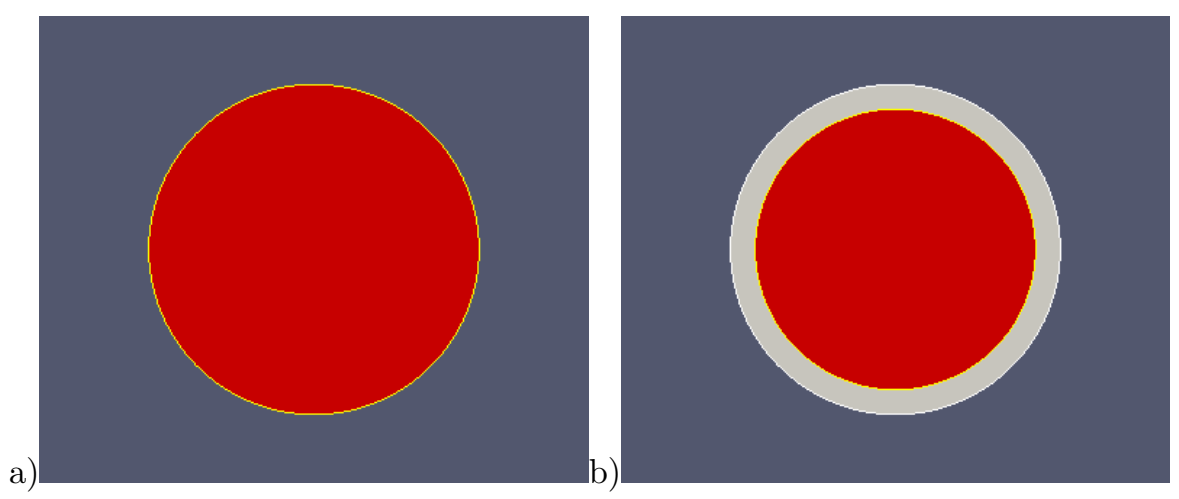

Figure 13: Geometry (IV): a) section $S\left(x_{1}\right)$ for $x_{1} \in(0,0.4) \cup(0.6,1)$, b) section $S\left(x_{1}\right)$ for $x_{1} \in(0.4,0.6)$. 


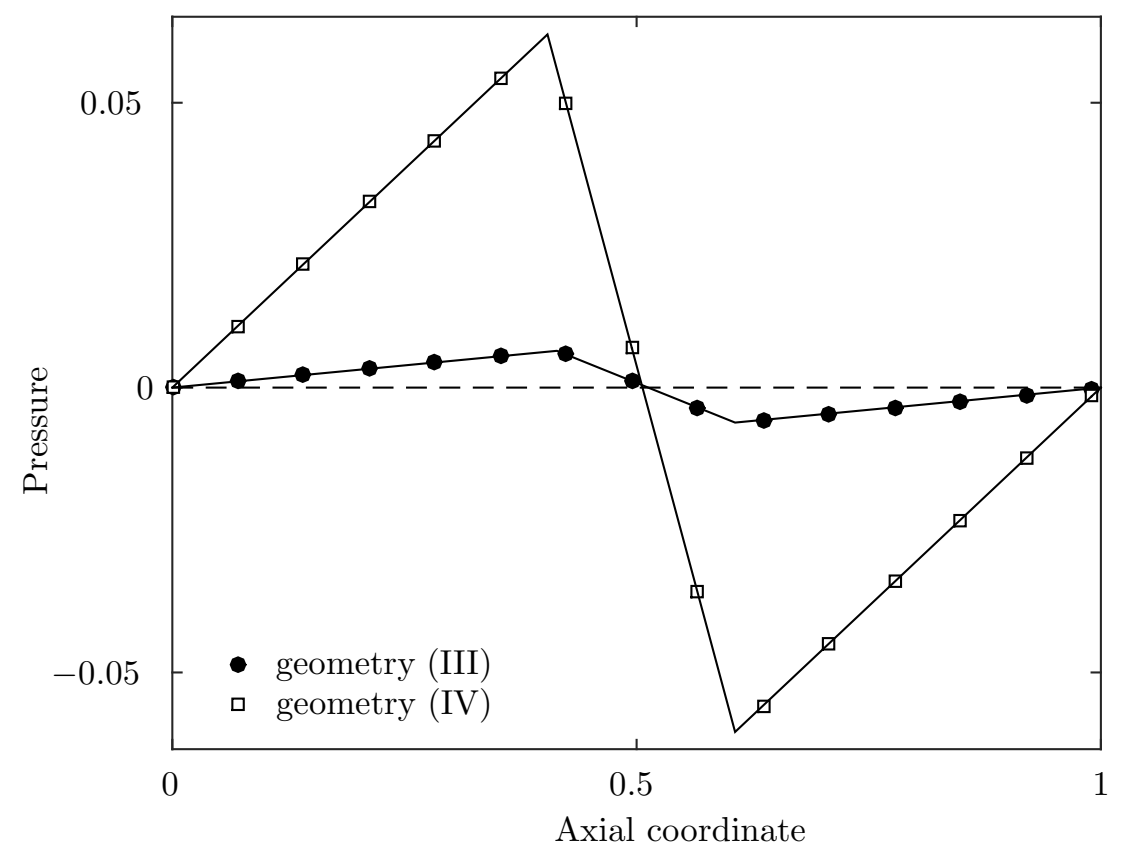

Figure 14: Geometries (III) and (IV): pressure profile in the asymptotic case (note that the linear contribution $x \mapsto-x$ has been removed).

- Figures 17 and 18 present the velocity and the components of the elastic stress tensor at $x_{1}=0.25$ (in the regular area) and $x_{1}=0.50$ (in the constricted area) for geometry (IV). Note that the velocity magnitude is more important in the constricted area, as it is expected.

The regular sections of geometries (III) and (IV) are the same: this implies that, considering the construction of the asymptotic model, the flux $\int_{S\left(x_{1}\right)} \tilde{u}_{1}^{0}$ through each section $S\left(x_{1}\right)$ in geometry (III) only differs from the one in geometry (IV) through the ratio between the derivative of their respective pressure distribution. As a consequence, denoting $F_{(\text {III })}$ and $F_{(\mathrm{IV})}$ the respective fluxes, we expect to obtain $F_{(\mathrm{III})}>F_{(\mathrm{IV})}$ (see the behaviour of the pressure derivative in geometries (III) and (IV) in Figure 14). In practical computations, we obtain $F_{(\mathrm{III})}=0.7436$ and $F_{(\mathrm{IV})}=0.6421$. 

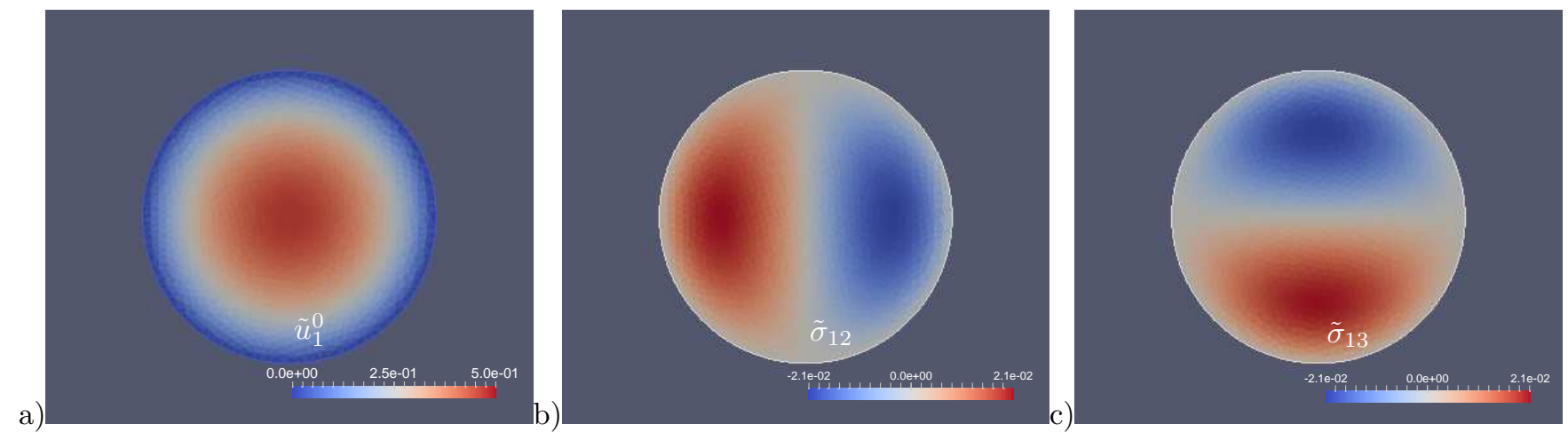

Figure 15: Geometry (III): normalized fields at $x_{1}=0.25$ : a) velocity profile $\tilde{u}_{1}^{0}$, b) extra-stress $\tilde{\sigma}_{12}$, c) extra-stress $\tilde{\sigma}_{13}$.
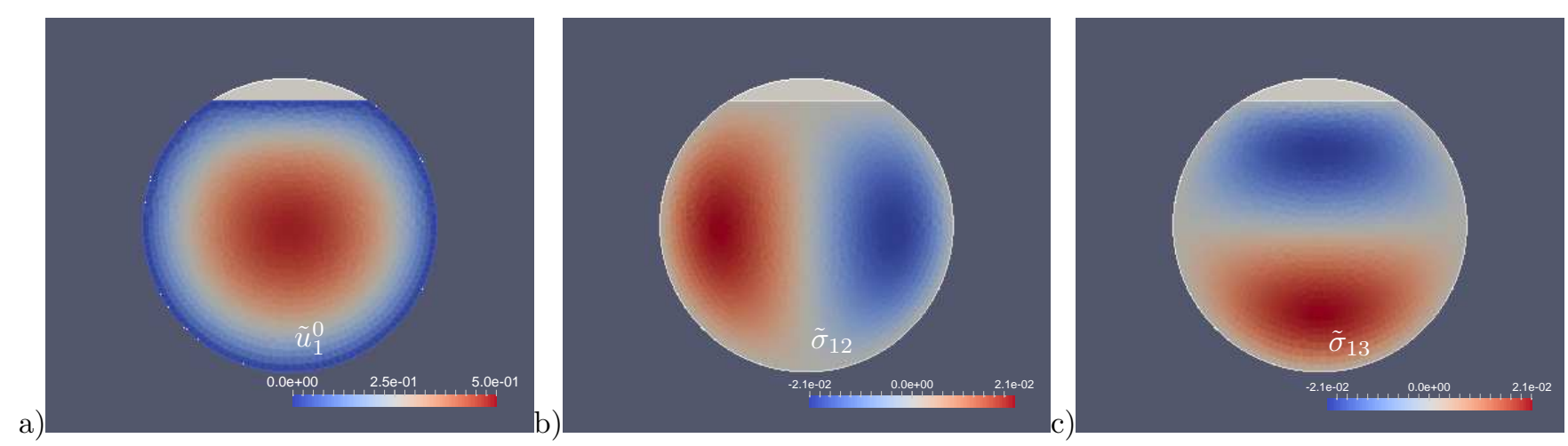

Figure 16: Geometry (III): normalized fields at $x_{1}=0.50$ : a) velocity profile $\tilde{u}_{1}^{0}, \mathrm{~b}$ ) extra-stress $\tilde{\sigma}_{12}, \mathrm{c}$ ) extra-stress $\tilde{\sigma}_{13}$. 

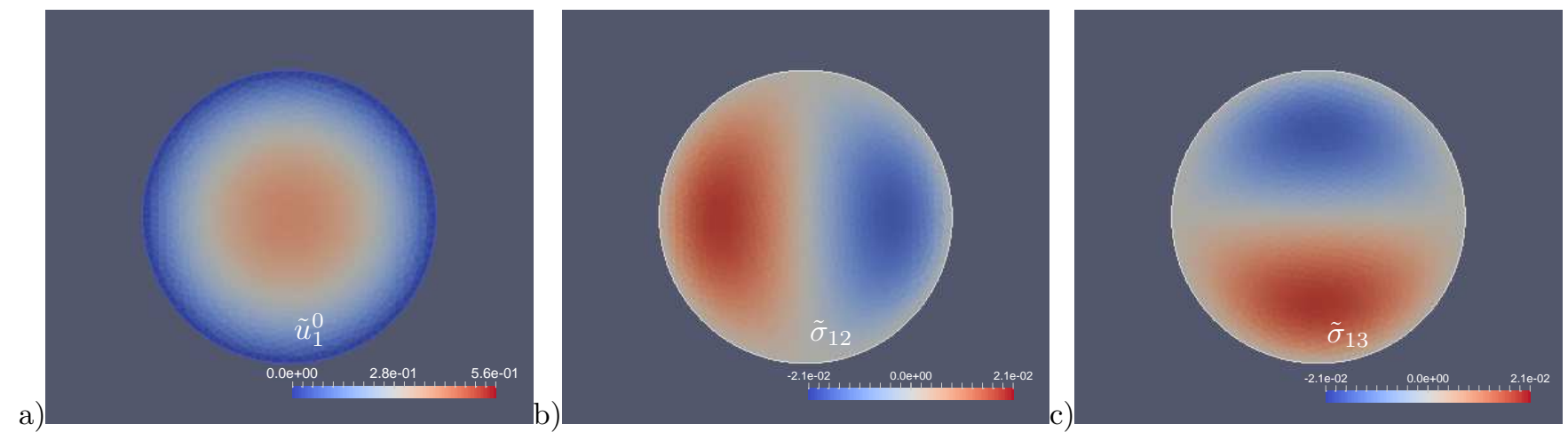

Figure 17: Geometry (IV): normalized fields at $x_{1}=0.25$ : a) velocity profile $\tilde{u}_{1}^{0}$, b) extra-stress $\tilde{\sigma}_{12}$, c) extra-stress $\tilde{\sigma}_{13}$.
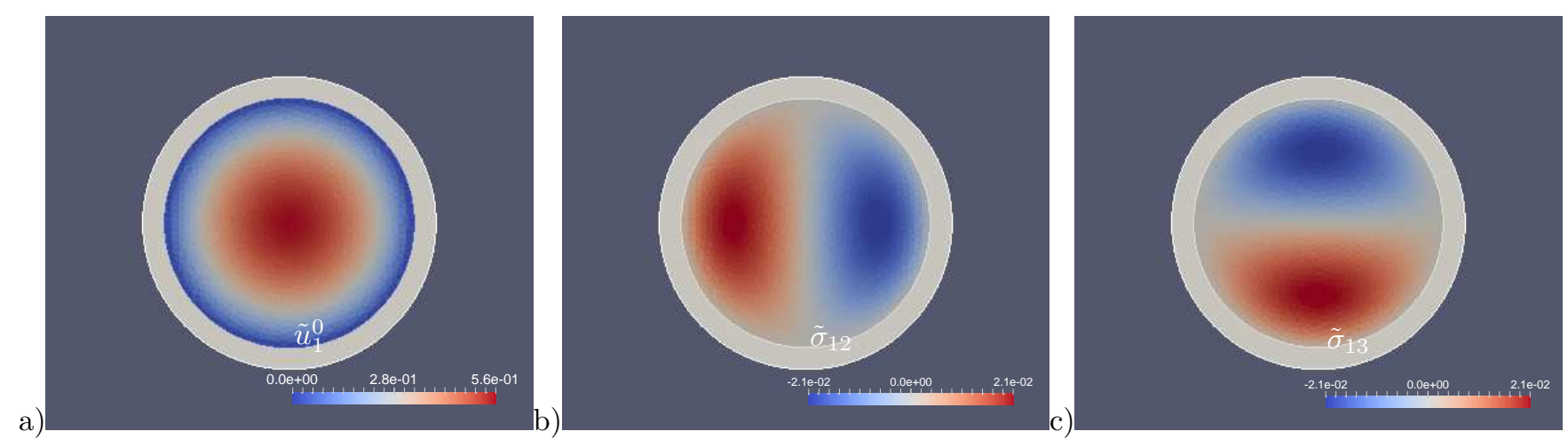

Figure 18: Geometry (IV): normalized fields at $x_{1}=0.50$ : a) velocity profile $\tilde{u}_{1}^{0}, \mathrm{~b}$ ) extra-stress $\tilde{\sigma}_{12}, \mathrm{c}$ ) extra-stress $\tilde{\sigma}_{13}$. 


\section{References}

[1] C. Bernardi, M. Dauge, Y. Maday, Spectral methods for axisymmetric domains, Series in Applied Mathematics, Gauthier-Villars, 345 pp., 1999.

[2] H. Brezis, Functional analysis, Sobolev spaces and partial differential equations, Springer, New York, 599 pp., 2011.

[3] S. Deparis, Numerical analysis of axisymmetric flows and methods for fluid-structure interaction arising in blood flow simulation, Thèse de l'École polytechnique fédérale de Lausanne, 2004.

[4] G.P. Galdi, An introduction to the mathematical theory of the Navier-Stokes equations. Steady-state problems, Second edition. Springer Monographs in Mathematics, Springer, New York, 1018 pp., 2011.

[5] V. Girault, P.-A. Raviart, Finite element methods for Navier-Stokes equations. Theory and algorithms. Springer Series in Computational Mathematics, Springer-Verlag, Berlin, 374 pp., 1986.

[6] A. Ichim, Asymptotic behaviour of a class of incompressible, quasi-Newtonian fluids in thin pipes, Asymptot. Anal., 95 (3-4), 325-344 (2015).

[7] J.-L. Lions, Quelques méthodes de résolution des problèmes aux limites non linéaires, Gauthier-Villars, 554 pp., 1969.

[8] J.G. Liu, W.C. Wang, Characterization and regularity for axisymmetric solenoidal vector fields with application to Navier-Stokes equation, SIAM J. Math. Anal, 5, 1825-1850, 2009.

[9] O. Gipouloux, E. Mariušić-Paloka, Asymptotic behaviour of the incompressible Newtonian flow through thin constricted fracture, Multiscale problems in science and technology (Dubrovnik, 2000), 189-202, Springer, Berlin, 2002.

[10] J. Nečas, Direct methods in the theory of elliptic equations, Springer Monographs in Mathematics. Springer, Heidelberg, 372 pp., 2012.

[11] G.P. Panasenko, Asymptotic expansion of the solution of Navier-Stokes equation in tube structure and partial asymptotic decomposition of the domain, Appl. Anal., 76 (3-4), 363-381, 2000.

[12] M. Renardy, Existence of slow steady flows of viscoelastic fluids with differential constitutive equations, Z. Angew. Math. Mech. 65 (9), 449-451, 1985. 